\title{
Analog Network Coding in the Multiple Access Relay Channel: Error Rate Analysis and Optimal Power Allocation
}

\begin{abstract}
In this paper, we consider Analog Network Coding (ANC) in the Multiple Access Relay Channel (MARC) with multiple relays, and provide the following three-fold contribution: 1) We introduce a tractable mathematical framework for computing the Symbol Error Rate (SER) of Maximum-Likelihood (ML), Zero-Forcing (ZF), and Minimum Mean Square Error (MMSE) receivers; 2) By capitalizing on this tractable mathematical framework, we formulate a power allocation problem that is proved to be convex for $\mathrm{ML}, \mathrm{ZF}$ and MMSE receivers; and 3) We provide closed-form expressions of the optimal power to be allocated to the sources and the relays for $\mathrm{ZF}$ and MMSE receivers. With the aid of Monte Carlo simulations, we validate the accuracy of the proposed mathematical framework for various network topologies and channel conditions, as well as study the effectiveness of optimal power allocation. It is shown, in particular, that power optimization is beneficial as the number of sources increases and if the quality of the source-relay links is better than the quality of the relay-destination links.
\end{abstract}

Index Terms-Analog Network Coding, Multiple Access Relay Channel, Maximum-Likelihood, Zero-Forcing, Minimum Mean Square Error.

\section{INTRODUCTION}

Network Coding (NC) was introduced as a method for achieving the maximum information flow in networks with multiple nodes, by enabling intermediate nodes to perform coding operations on the incoming packets, such as the exclusive-OR operation [1]. Although initially aimed for wired networks, the adoption of $\mathrm{NC}$ was subsequently extended to wireless networks that consist of intermediate nodes between the sources and the destination, such as relays [2].

Among the several $\mathrm{NC}$ techniques proposed in the literature [3]-[7] (see also references therein), various researchers have shown that Analog Network Coding (ANC) provides a good trade-off in terms of computational complexity and achievable performance [8], [9]. In ANC, in fact, the relays exploit the broadcast nature of the wireless channel by simply amplifying and forwarding a superposition of the received signals to them. Hence, ANC is considered to be an attractive option for future relay-based systems, especially when the relays are batterypowered terminals and, as a consequence, relaying operations should be kept as simple as possible. Since ANC does not need decoding at the relays, it requires less computational complexity than its digital counterparts (e.g., decode-and-forward and compute-and-forward). The price to be paid, however, is some performance degradation at low-SNR (Signal-to-Noise-Ratio) due to the noise amplification effect originating from using a non-regenerative transmission protocol. For this reason, ANC is considered to be a good candidate transmission technology at high-SNR, where the impact of noise amplification is less pronounced.

As far as the deployment of relays in real-world scenarios is concerned, a practical reference scenario considered in wireless standards [10] is the so-called Multiple Access Relay Channel (MARC) [6], where multiple sources communicate to a common destination through the use of one or multiple relays. Such a scenario finds practical applications for uplink transmission in cellular networks. Recently, several research works have investigated the achievable performance of ANC in the MARC, especially from an information-theoretic point of view [11]-[14] (and references therein). In particular, the authors of [11] prove that ANC achieves the optimal diversitymultiplexing tradeoff at high multiplexing gains, as well as that it outperforms the compress-and-forward and decodeand-forward relaying at low and high multiplexing gains, respectively. In [12], a family of optimal distributed spacetime codes [15] for application to the two-user MARC is proposed, which achieves the optimal diversity-multiplexing tradeoff. In [13], the authors characterize the optimal relay amplification factor and the resulting optimal rate regions in multihop MARCs. Finally, in [14] expressions of the outage probability and ergodic capacity are obtained when distributed relay selection is applied.

As mentioned above, ANC is considered to be a promising relaying protocol for transmission at high-SNR. In this operating regime, the communication-theoretic performance of cooperative relaying protocols are often quantified in terms of coding gain and diversity order [16]. Recently, the authors of [17] have studied these performance metrics in an attempt to quantify the potentials of ANC. In [17], in particular, a Maximum-Likelihood (ML) detector at the destination is considered and the diversity order of ANC by assuming best relay selection and distributed space-time coded transmission is studied. Motivated by the promising results in [17], in the present paper we are interested in studying the Symbol Error Rate (SER) and diversity order of low-complexity (linear) demodulators at the destination. The motivation and relevance of this analysis originates from the high computational complexity of ML demodulation for application to multi-source networks. In addition, we are interested in assessing the gains of optimal power allocation at sources and relays, which is not investigated in [17]. Since in some applications a feedback channel may not be available, in the present paper we focus our attention on a repetition-based relaying protocol. As a consequence, our study complements the analysis conducted in [17] for relay selection based protocols. The present paper is an extended version of [18], where linear receivers are not 
considered. For completeness, it is worth mentioning that SER and optimal power allocation of ANC have been studied in [19] by assuming repetition coding and ML detection. The analysis, however, is applicable to bidirectional communication protocols with only two sources and variable-gain relays. We focus our attention, on the other hand, on multiple-access channels with multiple sources and fixed-gain relays.

Contribution: The main technical contributions of the present paper can be summarized as follows:

1) A tractable mathematical framework for computing the SER of ML, Zero-Forcing (ZF), and Minimum Mean Square Error (MMSE) detectors [20] at the destination is introduced. The framework capitalizes on some high-SNR approximations, which make it suitable for analyzing the coding gain and diversity order. Closed-form expressions of the coding gain and diversity order are provided for the three demodulators. They highlight how the number of sources and relays affect the end-to-end performance. In particular, it is proved that the ratio of the coding gains of ZF and MMSE detectors is monotonic as a function of the number of sources, relays, and modulation order. The accuracy of the approximations is assessed with the aid of Monte Carlo simulations and a good match is shown.

2) By capitalizing on the closed-form expressions of the SER, an optimal power allocation problem is formulated aiming at identifying how to best split the available power budget between sources and relays. The optimization problem is proved to be convex for the three demodulators and, thus, to have a unique solution.

3) finally, a closed-form expression of the optimal powers is provided for ZF and MMSE detectors. With the aid of Monte Carlo simulations, it is shown that power optimization is beneficial as the number of sources increases and if the quality of the source-relay links is better than the quality of the relay-destination links.

Organization: The rest of the paper is organized as follows. In Section II, the system model is introduced. In Section III, high-SNR closed-form expressions of the SER are presented for ML, ZF and MMSE receivers, by assuming no direct links between the sources and the destination. In Section IV, we provide a SER expression for the ML detector and discuss why the corresponding SER expressions for the linear detectors cannot be obtained with the presented mathematical framework, when these links exist. In Section V, the optimal power allocation problem is formulated and proved to be convex for the examined receivers. Furthermore, its closedform solution is provided for ZF and MMSE detectors. In Section VI, numerical results are provided to substantiate mathematical derivations and findings. Finally, Section VII concludes this paper.

Notation: The following notation is used throughout this paper: i) $Q(x)=(1 / \sqrt{2 \pi}) \int_{x}^{\infty} \exp \left(-u^{2} / 2\right) d u$ denotes the Qfunction, ii) $E\{x\}$ denotes the mean value of the stochastic process $x$; iii) $|\cdot|$ and $\|\cdot\|_{F}^{2}$ denote the absolute value and Frobenius norm, respectively, iv) $K_{v}(\cdot)$ denotes the modified Bessel function of the second kind and of the $v_{t h}$ order, v) $E_{n}(x)=\int_{1}^{\infty} \frac{e^{-x t}}{t^{n}} d t$ denotes the exponential integral function, vi) $\log (\cdot)$ denotes the natural $\log$ arithm, vii) matrices and vectors are denoted in boldface, viii) (.) denotes the

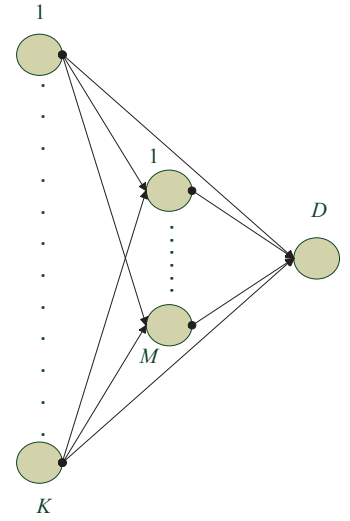

Fig. 1: The Multiple Access Relay Channel.

binomial coefficient, ix) $\operatorname{det}(\cdot)$ denotes the determinant of a matrix, $\mathrm{x}) e l_{p}(\cdot)$ takes as argument a diagonal matrix and denotes the elementary symmetric function [21] of order $p$ of the main diagonal elements of the matrix, and xi) $C N\left(\mu, \sigma^{2}\right)$ denotes a complex Gaussian random variable with mean equal to $\mu$ and variance equal to $\sigma^{2}$.

\section{SYSTEM MODEL}

We consider the MARC scenario depicted in Fig. 1, which may find application for uplink transmission in next generation cellular networks. In particular, $K$ sources want to communicate with a common destination through direct transmissions and $M$ fixed-gain and half-duplex relays. We, furthermore, assume that all the nodes are equipped with a single antenna and that the channel is narrowband block-fading. Let $\sigma_{k D}^{2}$, $\sigma_{k m}^{2}$, and $\sigma_{m_{D}}^{2}$ for $k=1,2, \ldots, K$ and $m=1,2, \ldots, M$ denote the path-loss coefficients of the source-destination, source-relay, and relay-destination links, respectively. They depend on the transmission distance between the nodes and the shadowing environment. Then, $h_{k D} \sim C N\left(0, \sigma_{k D}^{2}\right)$ denotes the channel coefficient from the $k_{t h}$ source to the destination, $h_{k m} \sim C N\left(0, \sigma_{k m}^{2}\right)$ denotes the channel coefficient from the $k_{t h}$ source to the $m_{t h}$ relay, and $f_{m} \sim C N\left(0, \sigma_{m_{D}}^{2}\right)$ denotes be the channel coefficient from the $m_{t h}$ relay to the destination. For mathematical tractability and for gaining design insights from the analysis, we assume, similar to [17], that $\sigma_{k D}^{2}=\sigma_{S D}^{2}, \sigma_{k m}^{2}=\sigma_{S R}^{2}$ and $\sigma_{m_{D}}^{2}=\sigma_{R D}^{2}$. Physically speaking, this means that the sources and the relays constitute two clusters (the nodes of the same cluster are located relatively close with each other in the same geographical area and, consequently, under the same shadowing effect) and, hence, the path-loss coefficients of the source-destination, sourcerelay and relay-destination links can be considered (almost) equal, respectively. Furthermore, we assume that: i) The channel coefficients that correspond to the source-destination, source-relay and relay-destination links are perfectly known to the destination (e.g., via pilot-based channel estimation prior to data transmission) together with the corresponding pathloss coefficients, ii) The relays have statistical knowledge of the source-relay links, which means that they are aware of $\sigma_{S R}^{2}$, and iii) No feedback channel exists in the system (open- 
loop system). Finally, to reduce the number of parameters for system design and optimization, we consider that the sources and the relays have equal transmission powers, $P_{S}$ and $P_{R}$, respectively, and that all the sources employ the same modulation order $Q$.

The communication protocol consists of two phases:

1st Phase - Transmission from the sources to the relays: During the first phase, which has a duration of 1 time slot, the $K$ sources simultaneously transmit their modulated packets $s_{k}$ to the destination and the relays. Hence, the received signal at the destination, which we denote as $y_{S D}$, is given by

$$
y_{S D}=\sqrt{P_{S} \sigma_{S D}^{2}} \sum_{k=1}^{K} \tilde{h}_{k D} s_{k}+n_{S D}
$$

where $\tilde{h}_{k D} \sim C N(0,1)$ and $n_{S D} \sim C N(0,1)$ denotes the Additive White Gaussian Noise (AWGN) at the destination during the 1st time slot. In addition, the received signal $y_{m}$ at the $m_{t h}$ relay is given by

$$
y_{m}=\sum_{k=1}^{K} \sqrt{P_{S} \sigma_{S R}^{2}} \tilde{h}_{k m} s_{k}+n_{m},
$$

where $\tilde{h}_{k m} \sim C N(0,1)$ and $n_{m} \sim C N(0,1)$ is the AWGN realization at the $m_{t h}$ relay.

This signal model assumes that there is perfect synchronization at the relays of the incoming signals from the sources, which can be achieved in practice by using the solutions in [8, Section 7.2]. The study of imperfect synchronization at the relays is an interesting future research direction.

2nd Phase - Transmission from the relays to the destination: During the second phase, the fixed-gain relays amplify their received signal and forward it to the destination. We consider a repetition-based transmission protocol from the relays, which implies that the relays sequentially forward their signal to the destination in $M$ non-overlapping time slots. Based on this protocol, $M+1$ time slots are required for the end-toend communication between the sources and the destination. Although repetition-based relaying is spectrally-inefficient, it constitutes a low-complexity option for leveraging the diversity potential of having multiple relays, especially in those application scenarios where relay selection protocols may not be used due to the unavailability of a feedback channel or when it may be too costly [17].

The gain $r_{m}$ of the relays, which normalizes their average transmission power with respect to the average received power of the sources, is given by [22]

$$
r_{m}=\sqrt{\frac{1}{K P_{S} \sigma_{S R}^{2}+1}} .
$$

Consequently, the received signal at the destination from each of the relays can be formulated as

$$
\begin{aligned}
y_{D_{m}} & =\sqrt{P_{R} \sigma_{R D}^{2}} r_{m} y_{m} \tilde{f}_{m}+n_{D_{m}} \\
& =\sqrt{\frac{P_{S} \sigma_{S R}^{2} P_{R} \sigma_{R D}^{2}}{K P_{S} \sigma_{S R}^{2}+1}} \tilde{f}_{m} \sum_{k=1}^{K} \tilde{h}_{k m} s_{k}+\tilde{n}_{D m},
\end{aligned}
$$

where $\tilde{f}_{m} \sim C N(0,1)$ and $n_{D_{m}} \sim C N(0,1)$ is the AWGN at the destination at each of the $M$ time slots of the relay transmission phase and $\tilde{n}_{D m} \sim C N\left(0, \frac{P_{R} \sigma_{R D}^{2}}{K P_{S} \sigma_{S R}^{2}+1}\left|\tilde{f}_{m}\right|^{2}+1\right)$.

In matrix form, by considering (1) and (4), the received signal vector, which we denote as $\mathbf{y}_{D \text {. Links, }}$ can be written as

$$
\begin{aligned}
& \mathbf{y}_{D . \text { Links }}=\left(\begin{array}{cccc}
y_{D_{1}} & \cdots & y_{D_{M}} & y_{S D}
\end{array}\right)^{T} \\
& =\sqrt{\frac{P_{S} \sigma_{S R}^{2} P_{R} \sigma_{R D}^{2}}{K P_{S} \sigma_{S R}^{2}+1}} \mathbf{F}_{\text {D. Links }} \mathbf{H}_{D . \text { Links }} \mathbf{s} \\
& +\tilde{\mathbf{n}}_{D_{D . \text { Links }}},
\end{aligned}
$$

where $\mathbf{F}_{\text {D. Links }} \in \mathbb{C}^{(M+1) \times(M+1)}$ is a diagonal matrix with $\tilde{f}_{1}, \ldots, \tilde{f}_{M}, \sqrt{P_{S} \sigma_{S D}^{2}} \sqrt{\frac{K P_{S} \sigma_{S R}^{2}+1}{P_{S} \sigma_{S R}^{2} P_{R} \sigma_{R D}^{2}}}$ being the elements of its main diagonal, $\mathbf{H}_{\text {D. Links }} \in \mathbb{C}^{(M+1) \times K}=$ $\left(\begin{array}{ccc} & \mathbf{H} & \\ \tilde{h}_{1 D} & \cdots & \tilde{h}_{K D}\end{array}\right)$, where $\mathbf{H} \in \mathbb{C}^{M \times K}$ with $\tilde{h}_{k m}$ as entries, $\mathbf{s}=\left(\begin{array}{ccc}s_{1} & \cdots & s_{K}\end{array}\right)^{T}$, and $\tilde{\mathbf{n}}_{D_{D . \text { Links }}}=$ $\left(\begin{array}{llll}\tilde{n}_{D_{1}} & \cdots & \tilde{n}_{D_{M}} & n_{S D}\end{array}\right)^{T}$.

Remark 1. From (5), it is clear that the MARC with ANC and time-orthogonal transmission from the relays is equivalent to a spatial multiplexing MIMO system [23] with channel matrix $\mathbf{F}_{\text {D. Links }} \mathbf{H}_{\text {D. Links }}$, where the sources and relays are the equivalent of transmit and receive antennas, respectively. However, there are two main differences with respect to a conventional direct-link MIMO system: i) The channel consists of the product of two matrices with random elements, in particular $\mathbf{F}_{\text {D. Links }}$ and $\mathbf{H}_{D \text {. Links }}$. ii) The noise is dependent on random variables, in particular the relay-to-destination channel gains. Consequently, due to the new statistics created by this type of MIMO channel compared to the direct-link MIMO counterpart, novel analytical expressions are needed to characterize the achievable error rate of ANC in the MARC. This is the main contribution of the present paper.

\section{Performance Analysis Assuming the Absence OF DIRECT LINKS}

In this section, we first derive approximate high-SNR closed-form formulas for the SER of ML, ZF, and MMSE receivers by assuming that $\sigma_{S D}^{2}=0$, i.e., no direct links are available. In practice, this can be attributed to a large distance or to a heavy-shadowing environment between the sources and the destination. The proposed mathematical expressions reveal the achievable coding gain and diversity order of each receiver. Subsequently, we prove the monotonic behavior of the coding gain ratio of $\mathrm{ZF}$ and $\mathrm{MMSE}$ receivers with respect to the number of sources, the number of relays, and the modulation order.

Let $\sigma_{S D}^{2}=0$. Then, the channel matrix is reduced to the matrix product $\mathbf{F H}$, where $\mathbf{F} \in \mathbb{C}^{M \times M}$ is a diagonal matrix with $\tilde{f}_{1}, \ldots, \tilde{f}_{M}$ being the elements of its main diagonal and $\mathbf{H}$ is defined in Section II. Consequently, the received signal 
vector, which we denote as $\mathbf{y}$, is given by

$$
\mathbf{y}=\sqrt{\frac{P_{S} \sigma_{S R}^{2} P_{R} \sigma_{R D}^{2}}{K P_{S} \sigma_{S R}^{2}+1}} \mathbf{F H s}+\tilde{\mathbf{n}}_{D}
$$

where $\tilde{\mathbf{n}}_{D}=\left(\begin{array}{lll}\tilde{n}_{D_{1}} & \cdots & \tilde{n}_{D_{M}}\end{array}\right)^{T}$.

\section{A. ML Detector}

By employing the ML-optimal detection criterion, the destination can jointly detect the transmitted symbols from the sources as

$$
\mathbf{s}_{d e t}=\arg \min _{\hat{s}_{k}} \sum_{m=1}^{M} \frac{\left|y_{D_{m}}-\sqrt{\frac{P_{S} \sigma_{S R}^{2} P_{R} \sigma_{R D}^{2}}{K P_{S} \sigma_{S R}^{2}+1}} \tilde{f}_{m} \sum_{k=1}^{K} \tilde{h}_{k m} \hat{s}_{k}\right|^{2}}{\frac{P_{R} \sigma_{R D}^{2}}{K P_{S} \sigma_{S R}^{2}+1}\left|\tilde{f}_{m}\right|^{2}+1},
$$

where $\mathbf{s}_{\text {det }}=\left(\begin{array}{lll}s_{1_{\text {det }}} & \cdots & s_{K_{\text {det }}}\end{array}\right)^{T}$ is the detected symbol vector.

Based on (7), we aim to derive an analytical closed-form framework for the union bound [23] of the SER per source. To this end, let $\left\{s_{q}\right\}$ denote the set of all possible $Q$ symbols transmitted from a particular source, which we assume to be equally probable. Furthermore, let $\{\mathbf{s}\}$ denote the set of the $Q^{K}$ symbol vectors to be transmitted form the $K$ sources, where $\left\{\mathbf{s}_{i}\right\}$ defines a subset of $\{\mathbf{s}\}$ in which the symbol vectors have $s_{q}$ transmitted from the $k_{t h}$ source. Thus, in total there are $Q^{K-1}$ such vectors. Also, let $\left\{\mathbf{s}_{j}\right\}$ denote the set of $Q^{K}-Q^{K-1}$ symbol vectors in which the symbol transmitted from the $k_{t h}$ source is different from $s_{q}$. Assuming that all the sources employ the same modulation order $Q$, the union bound of the SER for ML detection, which we denote as $S E R_{M L}$, is given by [23, Eq. (5)]

$$
S E R_{M L} \leqslant Q^{-K} \sum_{q=1}^{Q} \sum_{i=1}^{Q^{K-1}} \sum_{j=1}^{Q^{K}-Q^{K-1}} P E P_{s_{q}, i j}
$$

where $P E P_{s_{q}, i j}$ denotes the pairwise error probability (PEP) of detecting the symbol vector $\mathbf{s}_{j}$ when the vector $\mathbf{s}_{i}$ is transmitted.

Proposition 1. $P E P_{s_{q}, i j}$ can be approximated in closed-form as

$$
\begin{aligned}
P E P_{s_{q}, i j} & \approx \frac{1}{12}\left[\frac{1}{b+1}+\frac{b c}{a(b+1)^{2}} Z\left(\frac{c}{a(b+1)}\right)\right]^{M} \\
& +\frac{1}{6}\left[\frac{3}{4 b+3}+\frac{12 b c}{a(4 b+3)^{2}} Z\left(\frac{3 c}{a(4 b+3)}\right)\right]^{M},
\end{aligned}
$$

where $Z(x)=e^{x} E_{1}(x), a=P_{R} \sigma_{R D}^{2}, b=$ $\frac{1}{4} P_{S} \sigma_{S R}^{2}\left\|\boldsymbol{\Delta} \mathbf{s}_{i, j}\right\|_{F}^{2}$, where $\boldsymbol{\Delta} \mathbf{s}_{i, j}=\mathbf{s}_{i}-\mathbf{s}_{j}$, and $c=$ $K P_{S} \sigma_{S R}^{2}+1$.

Proof: See APPENDIX A.1.

By plugging (9) into (8), we obtain the union bound of the SER per source for ML detection.

Proposition 2. As $P_{S}, P_{R} \rightarrow \infty$, the pair-wise coding gain, which we denote as $G_{P_{a i r}{ }_{i j}}^{M L}$, and diversity order, which we denote as $G_{M L}^{d i v}$, of the ML detector are given by

$G_{\text {Pair }_{i j}}^{M L}=\left[\frac{1}{\left(\sigma_{R D}^{2}\left\|\boldsymbol{\Delta} \mathbf{s}_{i, j}\right\|_{F}^{2}\right)^{M}}\left[\frac{(4 K)^{M}}{12}+\frac{(3 K)^{M}}{6}\right]\right]^{-\frac{1}{M}}$,

$G_{M L}^{d i v}=M$.

\section{Proof: See APPENDIX A.2.}

Remark 2. From (10), we make the following two observations for the ML detector in the high-SNR regime: i) A full diversity order, equal to the number of relays, is achieved, regardless of the number of sources. ii) $G_{P a i r_{i j}}^{M L}$ (and, consequently, $S E R_{M L}$ ) decreases as the number of sources increases and, moreover, increases as $\sigma_{R D}^{2}$ increases. In particular, we observe that it only depends on $\sigma_{R D}^{2}$ and not on $\sigma_{S R}^{2}$. Hence, as $\sigma_{R D}^{2}$ increases, which means that the quality of the relay-destination links becomes better, $S E R_{M L}$ decreases.

\section{B. ZF Detector}

Due to the high complexity of the non-linear ML detector, even for a moderate number of sources and relays, it is of practical importance to consider low-complexity linear detectors that lead to individual source detection instead of a joint one, like the ML detector. Considering that the MARC with ANC is equivalent to a spatial multiplexing MIMO channel, as we showed in Section II, a simple detector that belongs to this category is the ZF detector [20]. In this case, the constraint $K \leqslant M$ needs to hold due to matrix inversion properties. Such a scenario requires an abundance of nodes acting as relays, which can be a real-world case considering that the relays can be inactive mobile terminals [28] or even the base stations of several underutilized femtocells overlaid in a macro-cell area with relaying capabilities [29], [30]. In the latter case, they can help, for instance, macro-cell users that are scheduled to be analog network-coded in the uplink.

With a $\mathrm{ZF}$ receiver and considering that the channel matrix is $\mathbf{F H}$, the ZF-equalized received signal vector $\mathbf{y}_{e q}^{Z F}$ is given by [20]

$$
\begin{aligned}
\mathbf{y}_{e q}^{Z F} \stackrel{(a)}{=} \mathbf{G}_{Z F} \mathbf{y} & =\sqrt{\frac{P_{S} \sigma_{S R}^{2} P_{R} \sigma_{R D}^{2}}{K P_{S} \sigma_{S R}^{2}+1}} \mathbf{s}+\mathbf{G}_{Z F} \tilde{\mathbf{n}}_{D} \\
& =\sqrt{\frac{P_{S} \sigma_{S R}^{2} P_{R} \sigma_{R D}^{2}}{K P_{S} \sigma_{S R}^{2}+1}} \mathbf{s}+\tilde{\mathbf{n}}_{Z F},
\end{aligned}
$$

where in $(a)$ we use (6) and, moreover,

$$
\mathbf{G}_{Z F}=\left[(\mathbf{F H})^{H} \mathbf{F H}\right]^{-1}(\mathbf{F H})^{H}, \quad \tilde{\mathbf{n}}_{Z F}=\mathbf{G}_{Z F} \tilde{\mathbf{n}}_{D} .
$$

Proposition 3. Let $K P_{S} \sigma_{S R}^{2}+1 \gg P_{R} \sigma_{R D}^{2}$. Then, an approximate expression in the high-SNR regime for the SER of the $\mathrm{ZF}$ detector, which we denote as $S E R_{Z F}$, is given by

$$
S E R_{Z F} \approx c_{1} A_{Z F}
$$


where

$$
\begin{aligned}
A_{Z F} & =\frac{1}{3}\left[\frac{1}{c_{2} \bar{\gamma}} e^{\frac{1}{c_{2} \bar{\gamma}}} E_{1}\left(\frac{1}{c_{2} \bar{\gamma}}\right)\right]^{M-K+1} \\
& +\frac{2}{3}\left[\frac{1}{c_{3} \bar{\gamma}} e^{\frac{1}{c_{3} \bar{\gamma}}} E_{1}\left(\frac{1}{c_{3} \bar{\gamma}}\right)\right]^{M-K+1}
\end{aligned}
$$

$\bar{\gamma}$ is the average pre-processing received SNR per source, given by

$$
\bar{\gamma}=\frac{P_{S} \sigma_{S R}^{2} P_{R} \sigma_{R D}^{2}}{K P_{S} \sigma_{S R}^{2}+P_{R} \sigma_{R D}^{2}+1},
$$

and

$$
c_{1}=1-\frac{1}{\sqrt{Q}}, \quad c_{2}=\frac{3}{2(Q-1)}, \quad c_{3}=\frac{2}{Q-1} .
$$

Proof: See APPENDIX A.3.

Proposition 4. As $\bar{\gamma} \rightarrow \infty$, the coding gain and diversity order of the ZF detector, which we denote as $G_{Z F}^{c o d}$ and $G_{Z F}^{d i v}$, respectively, are given by

$$
\begin{aligned}
G_{Z F}^{c o d} & =\left[\frac{c_{1}}{3}\left(c_{2}^{-(M-K+1)}+2 c_{3}^{-(M-K+1)}\right)\right]^{-\frac{1}{M-K+1}} \\
G_{Z F}^{d i v} & =M-K+1
\end{aligned}
$$

\section{Proof: See APPENDIX A.4.}

Remark 3. From (17), we observe that $G_{Z F}^{\text {div }}$ depends on the number of sources, as it was expected based on the ZF detector analysis in non-relay MIMO channels [20], and, specifically, it decreases as the number of sources increases. Hence, it is expected that the performance gap between the ML and ZF detectors increases as the number of sources increases since the diversity order of the former detector does not depend on the number of sources. In addition, we note that the Rayleighproduct nature of the MARC with ANC, according to (6), together with the approximation of (52), makes the derived SER expression for the ZF receiver of (13) valid also for the special category of channels that are called Rayleigh-product or multi-keyhole [33].

Remark 4. The SER approximate formula of (13) holds on the assumption that $K P_{S} \sigma_{S R}^{2}+1 \gg P_{R} \sigma_{R D}^{2}$. This condition is expected to hold, for example, in those scenarios where a large number of sources is available in the network and, thus, ZF detection may be a suitable alternative to ML detection because of the high computational complexity of the latter receiver. In Section VI, the accuracy of (13) is tested against Monte Carlo simulations and it is shown to be sufficiently accurate for $K P_{S} \sigma_{S R}^{2}+1>P_{R} \sigma_{R D}^{2}$ as well.

\section{MMSE Detector}

As in the ZF detector case, MMSE detection requires $K \leqslant$ $M$ to work. For a general channel matrix $\mathbf{A}$, the equalizing receive filter for the $k_{t h}$ source, which we denote as $\mathbf{g}_{M M S E, k}$ is given by $\left(\mathbf{A} \mathbf{A}^{H}+\frac{1}{\bar{\gamma}} \mathbf{I}_{M}\right)^{-1} \mathbf{a}_{k}$ [25], where $\mathbf{a}_{k}$ is the $k_{t h}$ column of $\mathbf{A}$. In our case, $\mathbf{A}=\mathbf{F H}$. Hence,

$$
\mathbf{g}_{M M S E, k}=\left(\mathbf{F H H}{ }^{H} \mathbf{F}^{H}+\frac{1}{\bar{\gamma}} \mathbf{I}_{M}\right)^{-1} \mathbf{F h}_{k},
$$

where $\bar{\gamma}$ is given by (15).

By considering (18), the MMSE-equalized received signal for the $k_{t h}$ source, which we denote as $y_{e q, k}^{M M S E}$, is given by

$$
\begin{aligned}
y_{e q, k}^{M M S E} & =\mathbf{g}_{M M S E, k}^{H} \mathbf{y} \\
& \stackrel{(f)}{=} \sqrt{\frac{P_{S} \sigma_{S R}^{2} P_{R} \sigma_{R D}^{2}}{K P_{S} \sigma_{S R}^{2}+1}} \mathbf{g}_{M M S E, k}^{H} \mathbf{F H s}+\mathbf{g}_{M M S E, k}^{H} \tilde{\mathbf{n}}_{D} \\
& =\sqrt{\frac{P_{S} \sigma_{S R}^{2} P_{R} \sigma_{R D}^{2}}{K P_{S} \sigma_{S R}^{2}+1}} \mathbf{g}_{M M S E, k}^{H} \mathbf{F} \mathbf{h}_{k} s_{k}+\tilde{n}_{k}^{M M S E},
\end{aligned}
$$

where in $(f)$ we use (6) and

$$
\tilde{n}_{k}^{M M S E}=\sum_{i \neq k} \mathbf{g}_{M M S E, k}^{H} \mathbf{F} \mathbf{h}_{i} s_{i}+\mathbf{g}_{M M S E, k}^{H} \tilde{\mathbf{n}}_{D} .
$$

As we observe from (20), $\tilde{n}_{k}^{M M S E}$ consists of the interference term $\sum_{i \neq k} \mathbf{g}_{M M S E, k}^{H} \mathbf{F} \mathbf{h}_{i} s_{i}$ and the noise term $\mathbf{g}_{M M S E, k}^{H} \tilde{\mathbf{n}}_{D}$.

Proposition 5. Let $K P_{S} \sigma_{S R}^{2}+1 \gg P_{R} \sigma_{R D}^{2}$. Then, an approximate expression in the high-SNR regime for the SER of the MMSE detector, which we denote as $S E R_{M M S E}$, is given by

$S E R_{M M S E} \approx \frac{c_{1}}{3}(K-1)\left(\begin{array}{c}M \\ M-K+1\end{array}\right)\left[A\left(c_{2}\right)+2 A\left(c_{3}\right)\right]$,

where $c_{1}, c_{2}$, and $c_{3}$ are given by (16), and

$$
\begin{aligned}
A(c) & =\left[\frac{1}{c \bar{\gamma}} e^{\frac{1}{c \bar{\gamma}}} E_{1}\left(\frac{1}{c \bar{\gamma}}\right)\right]^{M-K+1} \\
& \times e^{c} \sum_{k=0}^{K-2}(-1)^{k}\left(\begin{array}{c}
K-2 \\
k
\end{array}\right) E_{M-K+3+k}(c) .
\end{aligned}
$$

Proof: See APPENDIX A.5.

Proposition 6. As $\bar{\gamma} \rightarrow \infty$, the coding gain and diversity order of the MMSE detector, which we denote as $G_{M M S E}^{c o d}$ and $G_{M M S E}^{d i v}$, respectively, are given by

$$
G_{M M S E}^{c o d}=A_{M M S E}^{-\frac{1}{M-K+1}}, \quad G_{M M S E}^{d i v}=M-K+1,
$$

where

$$
A_{M M S E}=\frac{c_{1}}{3}(K-1)\left(\begin{array}{c}
M \\
M-K+1
\end{array}\right)\left[C\left(c_{2}\right)+2 C\left(c_{3}\right)\right]
$$

and

$$
C(c)=c^{-(M-K+1)} e^{c} \sum_{k=0}^{K-2}(-1)^{k}\left(\begin{array}{c}
K-2 \\
k
\end{array}\right) E_{M-K+3+k}(c) .
$$

\section{Proof: See APPENDIX A.6.}

Remark 5. From (23), we observe that the MMSE detector achieves the same diversity order as the ZF detector, but it has a different coding gain, as expected. Furthermore, similar comments as in Remark 3 and Remark 4 apply. 


\section{Comparison Between the ZF and MMSE Detection}

Since ZF and MMSE detectors achieve the same diversity order, as we have proved in Sections III-B and III-C, it is interesting to examine how the ratio of their coding gains, which we denote as $\frac{G_{M M S E}^{c o d}}{G^{c o d}}$, is affected by the number of sources, the number of relays, and the modulation order. From (17) and (23), we have

$\frac{G_{M M S E}^{c o d}}{G_{Z F}^{c o d}}=\left[\frac{c_{2}^{-(M-K+1)}+2 c_{3}^{-(M-K+1)}}{(K-1)\left(\begin{array}{c}M \\ M-K+1\end{array}\right)\left[C\left(c_{2}\right)+2 C\left(c_{3}\right)\right]}\right]^{\frac{1}{M-K+1}}$

Theorem 1. $\frac{G_{M M S E}^{c o d}}{G_{Z F}^{c o d}}$ is: i) A monotonically increasing function of $K$. ii) A monotonically decreasing function of $M$. iii) A monotonically decreasing function of $Q$.

Proof: See APPENDIX B.1.

Remark 6. These trends validate and, furthermore, corroborate the findings of [20], which was the first work presenting the surprising result that there is a non-vanishing gap between the performance of the $\mathrm{ZF}$ and MMSE receivers in a non-relay MIMO setup, as the SNR tends to infinity. Consequently, we have proved that the non-vanishing performance gap between these receivers behaves monotonically with respect to the number of sources, the number of relays, and the modulation order.

\section{Performance Analysis Assuming the Presence OF DIRECT LINKS}

In this section, the analysis of Section III is generalized by assuming the presence of direct links at the destination, i.e. $\sigma_{S D}^{2} \neq 0$. More specifically, we: i) Provide a closedform expression of the union bound of the SER per source for the ML receiver from which we extract its coding gain and diversity order. ii) Discuss why such a SER closed-form expression is difficult to obtain in the case of ZF and MMSE receivers.

Before proceeding with the analysis, we note that there are two differences with respect to the system model without direct links, i.e., $\sigma_{S D}^{2}=0$ : i) The last element $\sqrt{P_{S} \sigma_{S D}^{2}} \sqrt{\frac{K P_{S} \sigma_{S R}^{2}+1}{P_{S} \sigma_{S R}^{2} P_{R} \sigma_{R D}^{2}}}$ of $\mathbf{F}_{D . \text { Links }}$ (which corresponds to the direct links) does not have the same statistics as the other $M$ elements of $\mathbf{F}_{D \text {. Links }}$, in contrast to the matrix $\mathbf{F}$. ii) Accordingly, the last element of $\tilde{\mathbf{n}}_{D_{D \text {. Links }}}$ (which corresponds to the direct links) does not have the same statistics with the other $M$ elements of $\tilde{\mathbf{n}}_{D_{D \text {. Links }}}$.

\section{A. ML Detector}

By employing the ML detector, the detected symbol vector $\mathbf{s}_{\text {det }}=\left(\begin{array}{ccc}s_{1_{\text {det }}} & \cdots & s_{K_{\text {det }}}\end{array}\right)^{T}$ is given by

$$
\begin{aligned}
\mathbf{s}_{d e t} & =\arg \min _{\hat{s}_{k}}\left|y_{S D}-\sqrt{P_{S} \sigma_{S D}^{2}} \sum_{k=1}^{K} \tilde{h}_{k D} \hat{s}_{k}\right|^{2} \\
& +\sum_{m=1}^{M} \frac{\left|y_{D_{m}}-\sqrt{\frac{P_{S} \sigma_{S R}^{2} P_{R} \sigma_{R D}^{2}}{K P_{S} \sigma_{S R}^{2}+1}} \tilde{f}_{m} \sum_{k=1}^{K} \tilde{h}_{k m} \hat{s}_{k}\right|^{2}}{\frac{P_{R} \sigma_{R D}^{2}}{K P_{S} \sigma_{S R}^{2}+1}\left|\tilde{f}_{m}\right|^{2}+1} .
\end{aligned}
$$

According to (27) and by following the same analytical procedure as in Section III-A, an approximate expression for the PEP of the ML detector, which we denote as

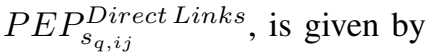

$$
\begin{aligned}
P E P_{s_{q, i j}}^{\text {Direct Links }} & =\frac{1}{12}\left[\frac{1}{b+1}+\frac{b c}{a(b+1)^{2}} Z\left(\frac{c}{a(b+1)}\right)\right]^{M} \\
& \times \frac{4}{4+P_{S} \sigma_{S D}^{2}\left\|\Delta \mathbf{s}_{i, j}\right\|_{F}^{2}} \\
& +\frac{1}{6}\left[\frac{3}{4 b+3}+\frac{12 b c}{a(4 b+3)^{2}} Z\left(\frac{3 c}{a(4 b+3)}\right)\right]^{M} \\
& \times \frac{3}{3+P_{S} \sigma_{S D}^{2}\left\|\Delta \mathbf{s}_{i, j}\right\|_{F}^{2}} .
\end{aligned}
$$

By plugging (28) into (8), we obtain the union bound of the SER per source of the ML detector with the inclusion of the direct links.

Proposition 7. Assuming that $P_{S}=P_{R} \rightarrow \infty$, the pair-wise coding gain, which we denote as $G_{P_{a i r}{ }_{i j}}^{M L-D i n k s}$, and diversity order, which we denote as $G_{M L-D}^{d i v}$. Links , of the ML detector are given by

$$
\begin{aligned}
& G_{\text {Pair }_{i j} . L^{2 i n k s}}^{M L-D}=A_{M L-D . L_{i n k s_{i, j}}}^{\frac{1}{M+1}}, \\
& G_{M L-D . \text { Links }}^{\text {div }}=M+1 .
\end{aligned}
$$

where

$$
\begin{aligned}
A_{M L-D . \text { Links }_{i, j}} & =\frac{1}{\left(\sigma_{R D}^{2}\right)^{M} \sigma_{S D}^{2}\left(\left\|\boldsymbol{\Delta} \mathbf{s}_{i, j}\right\|_{F}^{2}\right)^{M+1}} \\
& \times\left[\frac{4^{M+1} K^{M}}{12}+\frac{3^{M+1} K^{M}}{6}\right]
\end{aligned}
$$

Proof: It follows by using the same line of thought as the proof of Proposition 2.

Remark 7. From (29), we conclude that the inclusion of the direct links increases the diversity of the system by 1 , as expected.

\section{B. ZF and MMSE Detectors}

Following the same procedure, as in Sections III-B and III-C, the analysis of the SER of ZF and MMSE detectors involves the computation of $E\left\{\tilde{\mathbf{n}}_{D_{D . \text { Links }}} \tilde{\mathbf{n}}_{D_{D . \text { Links }}}^{H}\right\}$. We 
have

$$
\begin{aligned}
& E\left\{\tilde{\mathbf{n}}_{\left.D_{D . \text { Links }} \tilde{\mathbf{n}}_{D_{D . \text { Links }}}^{H}\right\}}\right. \\
& \stackrel{(q)}{\approx} \operatorname{diag}\left(\begin{array}{llll}
\frac{P_{R} \sigma_{R D}^{2}}{K P_{S} \sigma_{S R}^{2}+1} & \cdots & \frac{P_{R} \sigma_{R D}^{2}}{K P_{S} \sigma_{S R}^{2}+1} & 1
\end{array}\right),
\end{aligned}
$$

where $\operatorname{diag}(\cdot)$ denotes a diagonal matrix and in $(q)$ we use $K P_{S} \sigma_{S R}^{2}+1 \gg P_{R} \sigma_{R D}^{2}$. Consequently, $E\left\{\tilde{\mathbf{n}}_{D_{D \text {. Links }}} \tilde{\mathbf{n}}_{D_{D \text {. Links }}^{H}}^{S}\right\}$ does not have the same elements in the main diagonal (due to the last element that corresponds to the direct links) and, hence, convenient expressions such as (53) and (62) for the ZF and MMSE detectors, respectively, cannot be obtained. This issue, together with the fact that not all the elements of $\mathbf{F}_{D \text {. Links }}$ have the same statistics, creates the needs for new error rate frameworks that cannot be covered by the existing analysis. For this reason, this problem is left to future research activities.

\section{Optimal Power Allocation}

For a fair comparison among network topologies with a different number of sources and relays, it is common practice to compare them under two main assumptions: i) The rate (in bits) per channel use is the same and ii) the total power consumed in the networks, i.e., used by all the sources and the relays, is the same [19]. The first constraint is usually ensured by adapting the modulation order as a function of the used time slots. This is taken in due consideration in Section VI. The second constraint is usually ensured by assuming a total power budget in the network to be allocated to the sources and the relays. This needs to be taken in due consideration when formulating the optimal power allocation problem.

In particular, let $P_{\text {budget }}$ denotes the total power budget in the network. The aim of the optimal power allocation is to distribute $P_{\text {budget }}$ among these nodes in such a way that the SER per source is minimized. This is the main objective of this section. Due to space limitations, we consider only the case study for $\sigma_{S D}^{2}=0$. In particular, the optimal power allocation problem can be formulated as

$$
\begin{array}{ll}
\underset{P_{S}, P_{R}}{\operatorname{minimize}} & \operatorname{SER}\left(P_{S}, P_{R}\right) \\
\text { subject to } & P_{\text {budget }}=K P_{S}+M P_{R} .
\end{array}
$$

In the following, we: i) Prove that (32) is a convex problem for the three receivers under analysis and, consequently, it has a unique solution. ii) Provide its solution in closed form for the ZF and MMSE receivers.

\section{A. ML detector}

For the ML receiver, the PEP between the symbol vectors $\mathbf{s}_{i}$ and $\mathbf{s}_{j}$ is given by (9). To make the optimal power allocation problem analytically tractable, we consider the optimal power allocation in the high-SNR regime, i.e., we assume that
$P_{S}, P_{R} \gg 1$. Then, (9) can be upper bounded as

$$
\begin{aligned}
P E P_{s_{q}, i j} & \lesssim \frac{1}{\left[\left\|\Delta \mathbf{s}_{i, j}\right\|_{F}^{2}\right]^{M}}\left[\frac{(4 K)^{M}}{12}+\frac{(3 K)^{M}}{6}\right] \\
& \times\left[\frac{1}{\sigma_{S R}^{2}\left(P_{\text {budget }}-M P_{R}\right)}+\frac{\log \left(1+\sigma_{R D}^{2} P_{R}\right)}{\sigma_{R D}^{2} P_{R}}\right]^{M}
\end{aligned}
$$

where we have used $P_{S}=\frac{P_{\text {budget }}-M P_{R}}{K}$ and the inequality $E_{1}(z)<e^{-z} \log \left(1+\frac{1}{z}\right)^{K}[27, \quad 5.1 .20]$. As we observe from (33), the factor that is included in all the PEPs and depends on the power allocation is $\left[\frac{1}{\sigma_{S R}^{2}\left(P_{\text {budget }}-M P_{R}\right)}+\frac{\log \left(1+\sigma_{R D}^{2} P_{R}\right)}{\sigma_{R D}^{2} P_{R}}\right]^{M}$. Hence, the objective function of the SER bound to be included in the power allocation problem so as to minimize the SER is

$$
D_{M L}\left(P_{R}\right)=\frac{1}{\sigma_{S R}^{2}\left(P_{\text {budget }}-M P_{R}\right)}+\frac{\log \left(1+\sigma_{R D}^{2} P_{R}\right)}{\sigma_{R D}^{2} P_{R}} .
$$

Based on (34), in the case of ML detection (32) is formulated as follows:

$$
\begin{array}{ll}
\underset{P_{R}}{\operatorname{minimize}} & D_{M L}\left(P_{R}\right) \\
\text { subject to } & P_{R} \in\left(0, \frac{P_{\text {budget }}}{M}\right) .
\end{array}
$$

Theorem 2. (35) is a convex optimization problem in $P_{R} \in$ $\left(0, \frac{P_{\text {budget }}}{M}\right)$.

\section{Proof: See APPENDIX B.2.}

Due to the fact that the $D_{M L}\left(P_{R}\right)$ includes the term $\log \left(1+\sigma_{R D}^{2} P_{R}\right)$, it is not possible to find a closed-form expression for the value of $P_{R}$ that minimizes it. However, since $D_{M L}\left(P_{R}\right)$ is convex and a single-variable function, a line-search in any computational software program can be performed for finding this value. Subsequently, $P_{S}$ is obtained as $\frac{P_{b u d g e t}-M P_{R}}{K}$, for the given $P_{\text {budget }}$.

\section{B. $Z F$ detector}

From (13) and by using the inequality $E_{1}(z)<$ $e^{-z} \log \left(1+\frac{1}{z}\right)$ [27, 5.1.20], we get

$$
S E R_{Z F} \lesssim \frac{c_{1}}{3}\left[H\left(c_{2}\right)+2 H\left(c_{3}\right)\right],
$$

where

$$
H(c)=\frac{\log (1+c \bar{\gamma})}{c \bar{\gamma}}
$$

From (36), it follows that the objective function to be minimized is

$$
\begin{aligned}
D_{Z F}\left(P_{R}\right) & =\frac{\log (1+\bar{\gamma})}{\bar{\gamma}} \\
& \stackrel{(\underline{p})}{=} \frac{\log \left(1+\frac{\sigma_{S R}^{2} \sigma_{R D}^{2}}{K} \frac{\left(P_{\text {budget }}-M P_{R}\right) P_{R}}{\sigma_{S R}^{2} P_{\text {budget }}-P_{R}\left(M \sigma_{S R}^{2}-\sigma_{R D}^{2}\right)+1}\right)}{\frac{\sigma_{S R}^{2} \sigma_{R D}^{2}}{K} \frac{\left(P_{\text {budget }}-M P_{R}\right) P_{R}}{\sigma_{S R}^{2} P_{\text {budget }}-P_{R}\left(M \sigma_{S R}^{2}-\sigma_{R D}^{2}\right)+1}},
\end{aligned}
$$


where in $(p)$ we use $(15)$ and $P_{S}=\frac{P_{\text {budget }}-M P_{R}}{K}$. In addition, in (38) we have excluded the multiplicative factors $c_{2}, c_{3}>0$ that exist in (36) due to the fact that a $P_{R}$ value that minimizes the term $\frac{\log (1+\bar{\gamma})}{\bar{\gamma}}$ also minimizes $\frac{\log (1+c \bar{\gamma})}{c \bar{\gamma}} \forall c>0$, since $c$ is a multiplicative constant. Hence, for ZF detection (32) becomes

$$
\begin{array}{cl}
\underset{P_{R}}{\operatorname{minimize}} & R\left(P_{R}\right)=\frac{\log \left(1+S\left(P_{R}\right)\right)}{S\left(P_{R}\right)} \\
\text { subject to } & P_{R} \in\left(0, \frac{P_{\text {budget }}}{M}\right),
\end{array}
$$

where

$$
S\left(P_{R}\right)=\frac{\left(P_{\text {budget }}-M P_{R}\right) P_{R}}{\sigma_{S R}^{2} P_{\text {budget }}-P_{R}\left(M \sigma_{S R}^{2}-\sigma_{R D}^{2}\right)+1} .
$$

Now, we present Lemma 1, which will be used for proving Theorem 3 that is subsequently presented.

Lemma 1. Assuming two continuous functions $f(x)$ and $g(x)$, where $f(x)$ is concave and $g(x)$ is convex and nonincreasing (monotonically decreasing), it holds that $h(x)=$ $g(f(x))$ is a convex function.

Proof: See [36, (3.10)].

Theorem 3. $R\left(P_{R}\right)$ is a convex function in $P_{R} \in$ $\left(0, \frac{P_{\text {budget }}}{M}\right)$ and, hence, (39) is a convex optimization problem with solution $P_{R_{s o l}}$ given by (41) at the top of the next page.

Proof: See APPENDIX B.3.

\section{MMSE detector}

From (21) and by using $E_{1}(z)<e^{-z} \log \left(1+\frac{1}{z}\right)$ 5.1.20], we have

$S E R_{M M S E} \lesssim \frac{c_{1}}{3}(K-1)\left(\begin{array}{c}M \\ M-K+1\end{array}\right)\left[P\left(c_{2}\right)+P\left(c_{3}\right)\right]$,

where

$$
\begin{aligned}
P(c) & =\left[\frac{\log (1+c \bar{\gamma})}{c \bar{\gamma}}\right]^{M-K+1} \\
& \times e^{c} \sum_{k=0}^{K-2}(-1)^{k}\left(\begin{array}{c}
K-2 \\
k
\end{array}\right) E_{M-K+3+k}(c) .
\end{aligned}
$$

Due to fact that (42) contains the same term $\frac{\log (1+c \bar{\gamma})}{c \bar{\gamma}}, c>0$, that exists in the case of the $\mathrm{ZF}$ detector, the same optimal power allocation applies to the MMSE detector as the one for the $\mathrm{ZF}$ detector. Hence, $P_{R_{\text {sol }}}$ for the MMSE case is again given by (41).

\section{NumERICAL RESUlts}

Our aim in this section is threefold: i) To substantiate the close match, with respect to Monte Carlo simulations, of the derived analytical error rate expressions for the examined ML, $\mathrm{ZF}$, and MMSE receivers, ii) To study under which scenarios the optimal power allocation can be beneficial compared to equal power allocation, and iii) To numerically validate Theorem 1.

\section{A. SER Curves and Optimal Versus Equal Power Allocation} Policy

In Fig. 2 and Fig. 3, we illustrate the $S E R$ vs. $\frac{P_{\text {budget }}}{(K+M)}$ curves for the examined three receivers in balanced $\left(\sigma_{S R}^{2}=\right.$ $\left.\sigma_{R D}^{2}\right)$ and unbalanced $\left(\sigma_{S R}^{2} \neq \sigma_{R D}^{2}\right)$ scenarios and for a different number of sources. We have considered both the equal power allocation policy, denoted as EPA, and the optimal one, denoted as OPA, based on Section V. In addition, we have included the SER curves in the case where the direct links between the sources and the destination exist with $\sigma_{S D}^{2}=0.2$. From these figures, we observe the close match in the highSNR regime (equivalently, for SER $\leqslant 10^{-2}$, as we observe) of the derived analytical frameworks and the Monte Carlo simulations. The only exception is obtained for ZF and MMSE receivers when $K=2, \sigma_{S R}^{2}=1$ and $\sigma_{R D}^{2}=4$, for which it holds that $K P_{S} \sigma_{S R}^{2}+1<P_{R} \sigma_{R D}^{2}$. This looseness of the framework for this scenario was expected due to the approximation of (52). However, we note that the need for employing the suboptimal ZF and MMSE receivers becomes more relevant as $K$ increases, due to the high complexity of ML detection, and, hence, we expect that $K P_{S} \sigma_{S R}^{2}+1>$ $P_{R} \sigma_{R D}^{2}$ applies when these receivers need to be employed, which makes the derived SER frameworks for the ZF and MMSE receivers being in close agreement with the simulations results, as we observe in Fig. 3. Furthermore, we note that error rates lower or equal to $10^{-2}$ are typical error rate requirements from the system designer in uncoded systems (which is what we consider in our case), since with channel coding the error rates can be significantly reduced. Hence, although our derived SER formulas for the three examined receivers are based on high-SNR considerations, their close match with the Monte Carlo simulations for $\mathrm{SER} \leqslant 10^{-2}$ verifies their practical importance.

In addition, from Fig. 2 and Fig. 3 we observe the following regarding the optimal power allocation policy: i) The performance gains over the equal power allocation policy are practically the same for both the case where the optimal values of $P_{S}$ and $P_{R}$ are obtained analytically (Section $\mathrm{V}$ ) and iteratively through a linear search as Monte Carlo simulations are conducted (actual optimal power allocation). This validates the accuracy and importance of the optimal power allocation solution that was analytically derived in Section V. ii) The policy is more beneficial when $\sigma_{S R}^{2}>\sigma_{R D}^{2}$ (the quality of the source-relay links is better than the corresponding one of the relay-destination links). In particular, noticeable gains can be observed for this case. To understand why this happens, let us, for instance, examine the SER formula in the high-SNR regime of the ML receiver, according to (49). We see that when $P_{S}, P_{R} \rightarrow \infty$, the SER of the ML detector depends only on $P_{R}$ and $\sigma_{S R}^{2}$, which means that the higher these values are, the lower the SER is. Hence, if, for instance, $\sigma_{S R}^{2}>\sigma_{R D}^{2}$ and $P_{S}=P_{R}$ (EPA), the minimization of the SER per source entails allocating more power to the relays so that the factor $P_{R} \sigma_{R D}^{2}$ increases, based on (49). On the other hand, if $\sigma_{S R}^{2}<\sigma_{R D}^{2}$, then the factor $P_{R} \sigma_{R D}^{2}$ has an adequate value to give a low enough SER and, consequently, the optimal power allocation policy provides minimal gains, 


$$
P_{R_{\text {sol }}}= \begin{cases}\frac{M\left(1+\sigma_{S R}^{2} P_{\text {budget }}\right)-\sqrt{M^{2}\left(1+\sigma_{S R}^{2} P_{\text {budget }}\right)^{2}-M\left(M \sigma_{S R}^{2}-\sigma_{R D}^{2}\right)\left(P_{\text {budget }}+P_{\text {budget }}^{2} \sigma_{S R}^{2}\right)}}{M\left(M \sigma_{S R}^{2}-\sigma_{R D}^{2}\right)}, & \text { if } M \sigma_{S R}^{2} \neq \sigma_{R D}^{2} . \\ \frac{P_{\text {budget }}}{2 M}, & \text { otherwise. }\end{cases}
$$

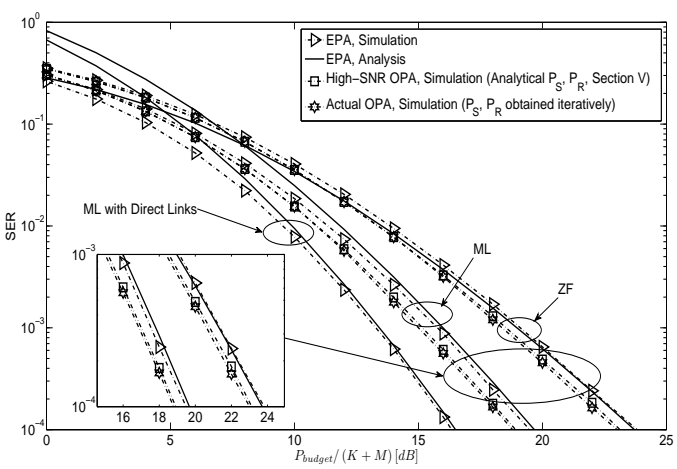

(a) ML and ZF for $\sigma_{S R}^{2}=4$ and $\sigma_{R D}^{2}=1$.

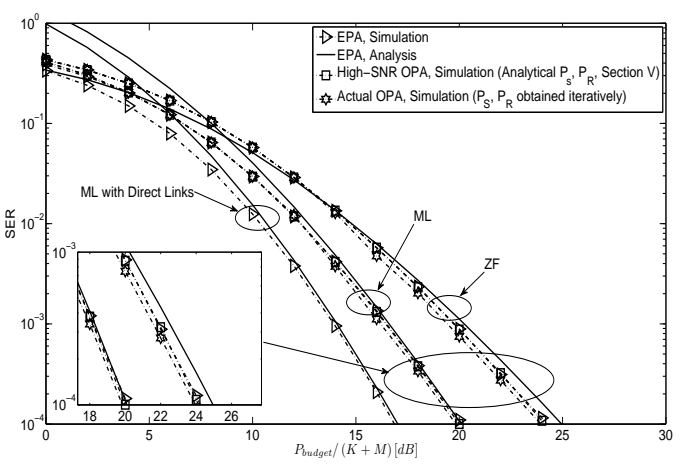

(c) $\mathrm{ML}$ and $\mathrm{ZF}$ for $\sigma_{S R}^{2}=1$ and $\sigma_{R D}^{2}=1$.

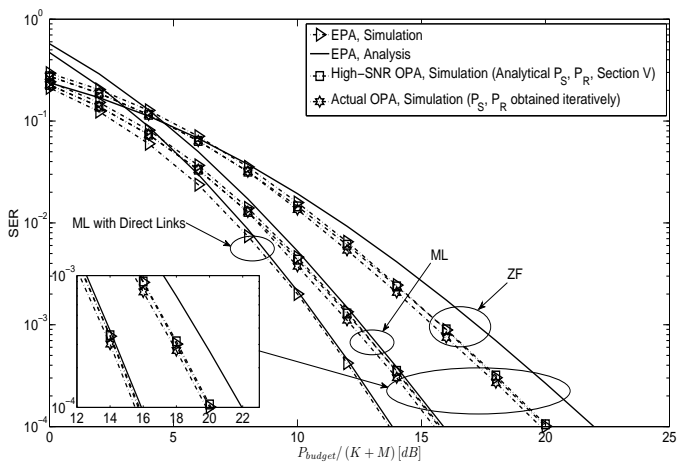

(e) ML and ZF for $\sigma_{S R}^{2}=1$ and $\sigma_{R D}^{2}=4$.

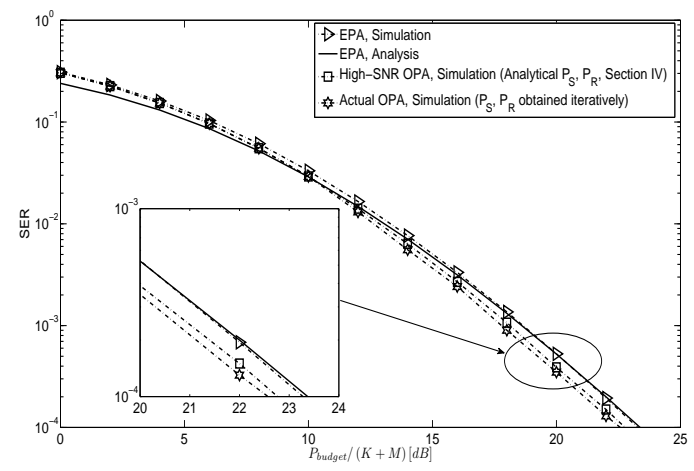

(b) MMSE for $\sigma_{S R}^{2}=4$ and $\sigma_{R D}^{2}=1$.

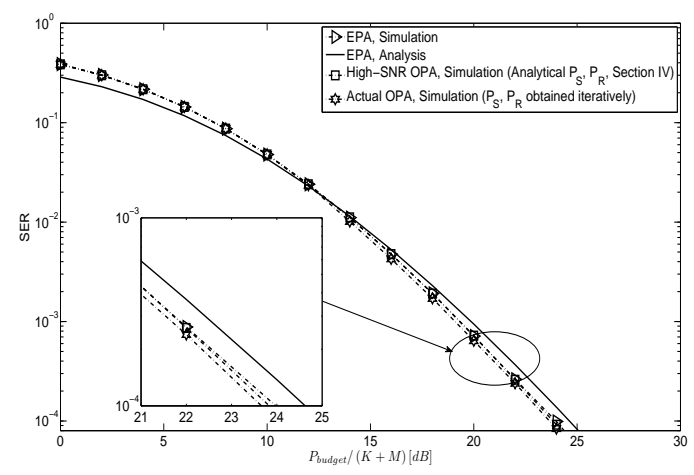

(d) MMSE for $\sigma_{S R}^{2}=1$ and $\sigma_{R D}^{2}=1$.

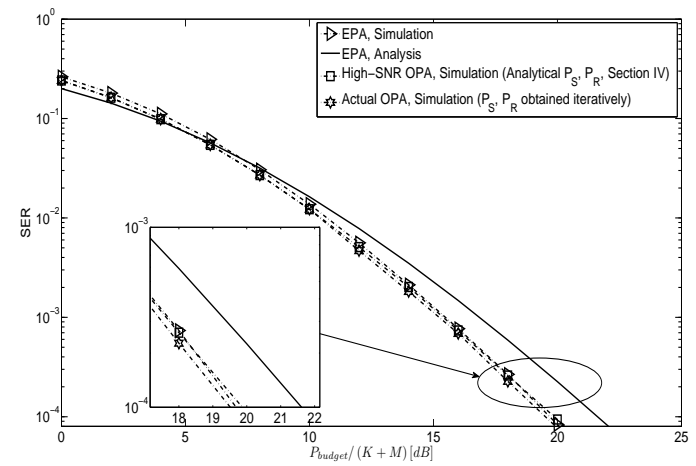

(f) MMSE for $\sigma_{S R}^{2}=1$ and $\sigma_{R D}^{2}=4$.

Fig. 2: SER vs. $\frac{P_{\text {budget }}}{(K+M)}[\mathrm{dB}]$ for $K=2, M=4$, and $Q=4$.

as we see in Fig. 2 and Fig. 3. A similar conclusion can be drawn by examining the high-SNR SER analytical error rate expressions of ZF and MMSE detectors. iii) The larger the number of sources is, the bigger the gain with respect to the equal power allocation policy is. This can be justified from the mathematical model and it can intuitively be explained by the fact that the sub-optimality of the latter policy becomes more pronounced as the number of nodes increases.

Now, in order to better understand the gains of the optimal power allocation with respect to the equal power allocation, which are observed in Fig. 2 and Fig. 3, we formulate the relative energy gain between these two policies, which we 


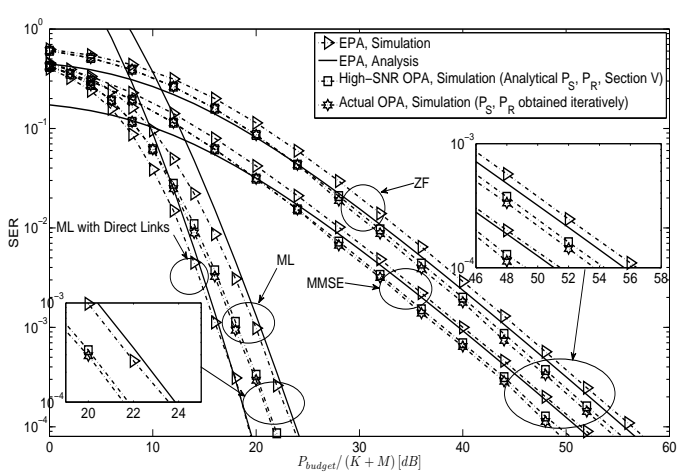

(a) ML, ZF, and MMSE for $\sigma_{S R}^{2}=4$ and $\sigma_{R D}^{2}=1$.

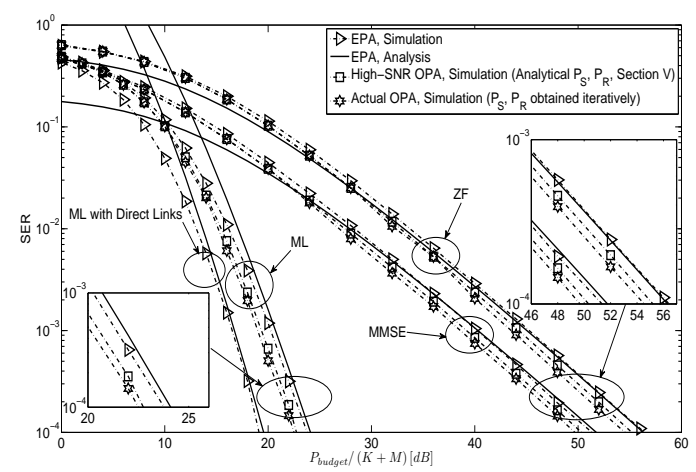

(b) ML, ZF, and MMSE for $\sigma_{S R}^{2}=1$ and $\sigma_{R D}^{2}=1$.

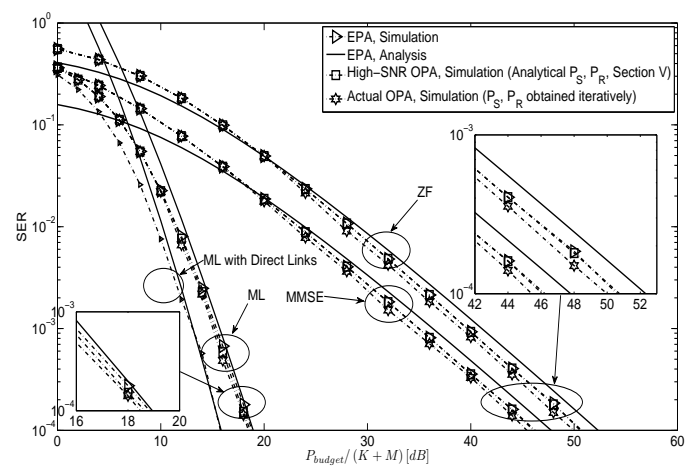

(c) ML, ZF, and MMSE for $\sigma_{S R}^{2}=1$ and $\sigma_{R D}^{2}=4$.

Fig. 3: SER vs. $\frac{P_{\text {budget }}}{(K+M)}[\mathrm{dB}]$ for $K=4, M=4$, and $Q=4$.

denote as $E_{\text {gain }}$, and it is given by

$$
E_{\text {gain }}=\left(P_{\text {buget }}^{E P A}-P_{\text {buget }}^{O P A}\right) / P_{\text {buget }}^{E P A}[\%],
$$

where $P_{\text {budget }}^{E P A}$ and $P_{\text {budget }}^{O P A}$ denote the total power budgets needed to achieve a target SER for the equal and optimal power allocation policies, respectively. Table I shows both the $E_{\text {gain }}$ and $P_{\text {budget }}^{E P A}-P_{\text {budget }}^{O P A}$ values with $P_{\text {budget }}^{E P A}$ and $P_{\text {budget }}^{O P A}$ obtained by using Monte Carlo simulations, for the examined configurations of Fig. 2 and Fig. 3 and for a target $S E R=10^{-4}$. For the optimal power allocation we used as input the values obtained by the solution the optimization problem presented in Section V.

As we observe from Table I, for $K=M=4, \sigma_{S R}^{2}=4$, and $\sigma_{R D}^{2}=1, E_{\text {gain }}$ is close to $37 \%$, which clearly indicates how important the optimal power allocation is for some configurations. Moreover, we observe that approximately the same gains are achieved with the optimal power allocation over the equal one for all the examined receivers. This constitutes an indication that the approximation of (52), which was used in the performance analysis of both the ZF and MMSE detector, does not lead to a suboptimal power allocation in the highSNR regime.

Finally, we note that the simulation results show that the performance gap between the ML and ZF/MMSE detectors increases by increasing the number of sources, which was expected, as we aforementioned in Section III-B, due to the dependency of the diversity order of the latter receivers on the number of sources in contrast to the ML detector.

In order to get some insights on how the power is allocated to the sources and the relays in the OPA case, in Table II we show $P_{S}$ an $P_{R}$ in the EPA and OPA cases for a $P_{\text {budget }}$ that gives a SER of $10^{-4}$ in the EPA case, $K=M=4$, and for balanced and unbalanced settings. As we observe from Table II, when the channel quality of the source-relay links is better or the same as that of the relay-destination links, notably less power should be allocated to the sources than to the relays for minimizing the SER. This is an important result from a practical point of view since the sources are likely to be battery-powered mobile terminals in the uplink and, hence, significant energy gains and, consequently, prolongation of operational duration for those devices, are expected for particular configurations by employing the OPA policy. On the other hand, although the power of the relays needs to increase in most of the examined scenarios compared to the EPA case, this may not pose a problem since we note that the relays are likely to be connected to the power network (since it is more likely to be larger devices, as discussed in Section III-B).

\section{B. Numerical Validation of Theorem 1}

To numerically validate Theorem 1 , we consider the ratio $\frac{S E R_{Z F}}{S E R_{M M S E}}$ of the SER of the ZF and MMSE detectors. In Fig. 4, we show how $\frac{S E R_{Z F}}{S E R_{M M S E}}$ is affected by $K, M$, and 
TABLE I: $P_{\text {budget }}^{E P A}-P_{\text {budget }}^{O P A}$ and $E_{\text {gain }}$ for $M=4, Q=4$, and target SER $=10^{-4}$.

\begin{tabular}{|c|c|c|c|c|c|}
\hline$\sigma_{S R}^{2}=4$ and $\sigma_{R D}^{2}=1$ & $\sigma_{S R}^{2}=1$ and $\sigma_{R D}^{2}=1$ & $\sigma_{S R}^{2}=1$ and $\sigma_{R D}^{2}=4$ \\
\hline ML & ZF/MMSE & ML & ZF/MMSE & ML & ZF/MMSE \\
\hline \multicolumn{7}{|c|}{$P_{\text {budget }}^{\text {EPA }}-P_{\text {budget }}^{\text {OPA }}[\mathrm{dB}] \& E_{\text {gain }}(\%)$} \\
\hline \multicolumn{7}{|c|}{$K=2$} \\
\hline $0.7 \& 14.9$ & $0.7 \& 14.9$ & $0 \& 0$ & $0 \& 0$ & $0 \& 0$ & $0 \& 0$ \\
\hline \multicolumn{7}{|c|}{$K=3$} \\
\hline $1.5 \& 29$ & $1.4 \& 27.6$ & $0.7 \& 14.9$ & $0.7 \& 14.9$ & $0.1 \& 2.3$ & $0 \& 0$ \\
\hline \multicolumn{7}{|c|}{$K=4$} \\
\hline $2 \& 36.9$ & $2 \& 36.9$ & $1.2 \& 24.1$ & $1 \& 20.1$ & $0.2 \& 4.5$ & $0 \& 0$ \\
\hline
\end{tabular}

TABLE II: EPA and OPA (in $\mathrm{dB}$ ) for $K=M=4, Q=4$, and $P_{\text {budget }}$ that gives $\mathrm{SER}=10^{-4}$ in the EPA case.

\begin{tabular}{|c|c|c|c|c|c|}
\hline \multicolumn{2}{|c|}{ ML } & ZF & \multicolumn{2}{c|}{ MMSE } \\
\hline EPA & OPA & EPA & OPA & EPA & OPA \\
\hline \multicolumn{7}{|c|}{$\sigma_{S R}^{2}=4$ and $\sigma_{R D}^{2}=1$} \\
\hline$P_{S}=P_{R}=23.8$ & $\begin{array}{l}P_{S}=16.8 \\
P_{R}=26.4\end{array}$ & $P_{S}=P_{R}=56$ & $\begin{array}{c}P_{S}=52 \\
P_{R}=58\end{array}$ & $P_{S}=P_{R}=51.5$ & $\begin{array}{c}P_{S}=47.5 \\
P_{R}=53.5\end{array}$ \\
\hline \multicolumn{7}{|c|}{$\sigma_{S R}^{2}=1$ and $\sigma_{R D}^{2}=1$} \\
\hline$P_{S}=P_{R}=24.1$ & $P_{S}=19.7$ & $P_{S}=P_{R}=56.3$ & $\begin{array}{c}P_{S}=54.5 \\
P_{R}=57.5\end{array}$ & $P_{S}=P_{R}=51.8$ & $P_{S}=50$ \\
& $P_{R}=26.2$ & $\sigma_{S R}=1$ and $\sigma_{R D}^{2}=4$ \\
\hline \multicolumn{7}{|c|}{$P_{S}^{2}=P_{R}=22$} & $P_{S}=19.7$ & $P_{S}=P_{R}=52$ & $\begin{array}{c}P_{S}=52 \\
P_{R}=23.5\end{array}$ & \multirow{2}{*}{$P_{S}=P_{R}=48$} & $P_{S}=48$ \\
\hline
\end{tabular}

$Q$, by keeping two of these parameters fixed and varying the remaining one. We note that $S E R_{Z F}$ and $S E R_{M M S E}$ have been obtained by means of Monte Carlo simulations for the same $P_{S}$ and $P_{R}$. Furthermore, we have considered values of $P_{S}$ and $P_{R}$ that give $S E R_{Z F}, S E R_{M M S E}<10^{-4}$, since such low SER values correspond to the high-SNR regime, where the coding gain and diversity order are achieved.

As we observe from Fig. 4, $\frac{S E R_{Z F}}{S E R_{M M S E}}$ : i) Increases with increasing $K$. ii) Decreases with increasing $M$ and increasing $Q$. Consequently, these trends numerically validate Theorem 1 . From a practical point of view, the message that Fig. 4 conveys is that when $K$ is notably lower than $M$ or the modulation order is high $(Q>16$, for instance), it is practically not more beneficial to employ the MMSE receiver instead of the less complex ZF.

\section{CONCLUSIONS}

In this paper, we have analytically studied the error rate performance and optimal power allocation of ANC in the MARC. Three receiver schemes have been investigated (ML, $\mathrm{ZF}$ and MMSE) and their coding gains and diversity orders have been computed. These results show that the gap between the SER of the ML and the ZF/MMSE receivers increases as the number of sources increases. Moreover, monotonic trends for the ratio of the coding gains of $\mathrm{ZF}$ and MMSE receivers with respect to the number of sources, relays, and modulation order have been proved, which provide information on whether to employ MMSE detection or the less complex ZF, according to the system configuration. In particular, the gap between the SER of these two receivers becomes negligible if the number of relays is notably higher than the number of sources or the modulation order is high. Finally, we have proved the convexity of the optimal power allocation problem to minimize the SER per source of the examined receivers and, in addition, we have provided its closed-form solution for ZF and MMSE demodulators.

Noticeable energy gains of the optimal power allocation policy over the equal one are observed, as the number of sources increases and when the the quality of the source-relay links is better than that of the relay-destination links. On the other hand, when the relay-destination links have a better quality than the source-relay links, the optimal power allocation policy provides negligible gains. Moreover, the results show that the analytically derived optimal power allocation provides the same gains as the actual optimal power allocation, which substantiates the importance of our analysis. Finally, we have shown that in unbalanced scenarios, and, more specifically, when the quality of the source-relay links is better than that of the relay-destination links, the power allocated to the sources for SER minimization can be substantially lower than the one allocated to the relays. Hence, important energy gains for the sources can be achieved, which are likely to be batterypowered mobile terminals.

As future work, we aim to analytically study the error rate performance of ANC in the MARC by taking practical considerations into account, such as imperfect synchronization at the relays and outdated channel estimates at the destination. In addition, the analytical study of the ZF and MMSE receivers when the direct links are included in the system model is another interesting research direction. 


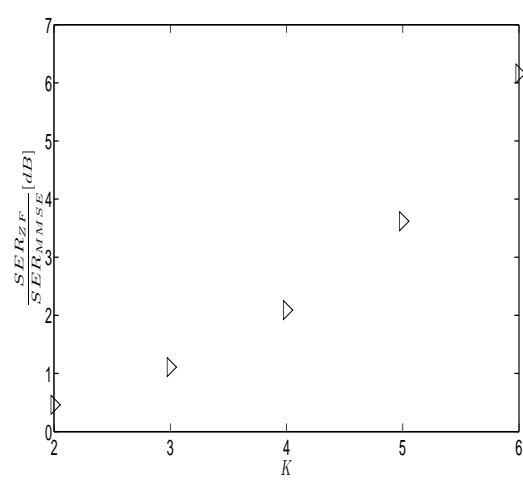

(a)

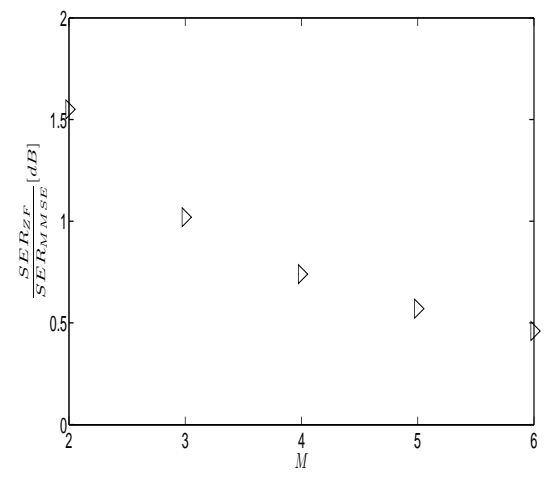

(b)

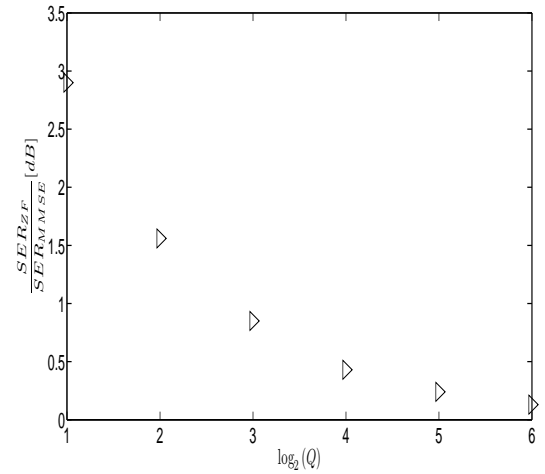

(c)

Fig. 4: $\frac{S E R_{Z F}}{S E R_{M M S E}}$ in the high-SNR regime $\left(S E R_{Z F}, S E R_{M M S E}<10^{-4}\right)$ vs. (a) $K$ for $M=6$ and $Q=4$, (b) $M$ for $K=2$ and $Q=4$, and (c) $\log _{2}(Q)$ for $K=M=2$. For all the plots, we used $\sigma_{S R}^{2}=\sigma_{R D}^{2}=1$.

\section{APPENDIX A}

A.1. Proof of Proposition 1: Based on (7), $P E P_{s_{q}, i j}$ is given by [24]

$$
\begin{aligned}
& P E P_{s_{q}, i j} \\
& =E\left\{Q\left(\sqrt{\sum_{m=1}^{M} \frac{1}{2} \frac{P_{S} \sigma_{S R}^{2} P_{R} \sigma_{R D}^{2}\left|\mathbf{h}_{m} \boldsymbol{\Delta} \mathbf{s}_{i, j}\right|^{2}\left|\tilde{f}_{m}\right|^{2}}{P_{R} \sigma_{R D}^{2}\left|\tilde{f}_{m}\right|^{2}+K P_{S} \sigma_{S R}^{2}+1}}\right)\right\},
\end{aligned}
$$

where $\mathbf{h}_{m}=\left(\begin{array}{lll}\tilde{h}_{1 m} & \tilde{h}_{2 m} \cdots \tilde{h}_{K m}\end{array}\right)$. Due to the difficulty of analytically solving (45) in its exact form, we consider the exponential approximation of the Q-function [25, Eq. (31)]:

$$
Q(x) \approx \frac{1}{12} e^{-\frac{x^{2}}{2}}+\frac{1}{6} e^{-\frac{2 x^{2}}{3}}, \quad x \geqslant 0 .
$$

By plugging (46) into (45) and by taking into account that all the channel links are independent, we obtain

$$
\begin{aligned}
P E P_{s_{q}, i j} & \approx \frac{1}{12} \prod_{m=1}^{M} \int_{0}^{\infty} e^{-u_{m}} f_{m}\left(u_{m}\right) d u_{m} \\
& +\frac{1}{6} \prod_{m=1}^{M} \int_{0}^{\infty} e^{-\frac{4}{3} u_{m}} f_{m}\left(u_{m}\right) d u_{m},
\end{aligned}
$$

where $u_{m}=\frac{1}{4} \frac{P_{S} \sigma_{S R}^{2} P_{R} \sigma_{R D}^{2}\left|\mathbf{h}_{m} \Delta \mathbf{s}_{i, j}\right|^{2}\left|\tilde{f}_{m}\right|^{2}}{P_{R} \sigma_{R D}^{2}\left|\tilde{f}_{m}\right|^{2}+K P_{S} \sigma_{S R}^{2}+1}$ and $f_{m}(u)$ is the probability density function of $u_{m}$. According to [22, Eq. (10)],

$f_{m}(u)=\frac{2}{b} e^{-\frac{u}{b}}\left[\sqrt{\frac{c u}{a b}} K_{1}\left(2 \sqrt{\frac{c u}{a b}}\right)+\frac{c}{a} K_{0}\left(2 \sqrt{\frac{c u}{a b}}\right)\right]$.

By plugging (48) into (47) and by using [26, 6.643.3] and [27, 13.1.33 and 13.2.5], we obtain (9).

A.2. Proof of Proposition 2: For $P_{S}, P_{R} \rightarrow \infty$ and by using the inequality $E_{1}(z)<e^{-z} \log \left(1+\frac{1}{z}\right)[27,5.1 .20]$ and the fact that $\log \left(\log \left(P_{R} \sigma_{R D}^{2}\right)\right) \ll \log \left(\stackrel{P}{P}_{R} \sigma_{R D}^{2}\right)$ for $P_{R} \rightarrow \infty$,
(9) is upper bounded as

$P E P_{s_{q}, i j} \lesssim \frac{1}{\left(\sigma_{R D}^{2}\left\|\Delta \mathbf{s}_{i, j}\right\|_{F}^{2}\right)^{M}}\left[\frac{(4 K)^{M}}{12}+\frac{(3 K)^{M}}{6}\right] P_{R}^{-M}$.

By considering that in the high-SNR regime the SER of an uncoded (or coded) system can be approximated as $S E R \approx$ $\left(G_{c} \bar{\gamma}\right)^{-G_{d}}$ [16], where $G_{c}, G_{d}$, and $\bar{\gamma}$ denote the coding gain, diversity order, and average SNR, respectively, the proof of Proposition 2 is concluded.

A.3. Proof of Proposition 3: The proof consists of the following two steps: i) We derive an approximate expression for the post-processing SNR of the $k_{t h}$ source, which we denote as $\gamma_{k}^{Z F}$. ii) Based on i), we obtain an approximate expression of the Moment Generating Function (MGF) of $\gamma_{k}^{Z F}$, which we denote as $M_{\gamma, k}^{Z F},(s)$ from which the SER per source of the ZF detector can be obtained as [25, Eq. (33)]

$$
S E R_{Z F} \approx c_{1}\left[\frac{1}{3} M_{\gamma, k}^{Z F}\left(c_{2}\right)+\frac{2}{3} M_{\gamma, k}^{Z F}\left(c_{3}\right)\right] .
$$

i) $\gamma_{k}^{Z F}$ : We first find the expected value of the covariance matrix of $\tilde{\mathbf{n}}_{Z F}$, conditioned on $\mathbf{H}$ and $\mathbf{F}$, which we denote as $E\left\{\tilde{\mathbf{n}}_{Z F} \tilde{\mathbf{n}}_{Z F}^{H} \mid \mathbf{H}, \mathbf{F}\right\}$. We have

$$
E\left\{\tilde{\mathbf{n}}_{Z F} \tilde{\mathbf{n}}_{Z F}^{H} \mid \mathbf{H}, \mathbf{F}\right\}=\mathbf{G}_{Z F} E\left\{\tilde{\mathbf{n}}_{D} \tilde{\mathbf{n}}_{D}^{H}\right\} \mathbf{G}_{Z F}^{H} .
$$

Now, for the term $E\left\{\tilde{\mathbf{n}}_{D} \tilde{\mathbf{n}}_{D}^{H}\right\}$ of (51), we have

$$
\begin{aligned}
E\left\{\tilde{\mathbf{n}}_{D} \tilde{\mathbf{n}}_{D}^{H}\right\} & =\left[\frac{P_{R} \sigma_{R D}^{2}}{K P_{S} \sigma_{S R}^{2}+1} \mathbf{V}+\mathbf{I}_{M}\right] \\
& \stackrel{(b)}{\approx}\left(\frac{P_{R} \sigma_{R D}^{2}}{K P_{S} \sigma_{R D}^{2}+1}+1\right) \mathbf{I}_{M},
\end{aligned}
$$

where $\mathbf{V}=\mathbf{F} \mathbf{F}^{H}=\mathbf{F}^{H} \mathbf{F}$ and the approximation in $(b)$ comes as a result of considering $K P_{S} \sigma_{S R}^{2}+1 \gg P_{R} \sigma_{R D}^{2}$. This is due to the fact that by this assumption the variance of $\frac{P_{R} \sigma_{R D}^{2}}{K P_{S} \sigma_{S R}^{2}+1}\left|\tilde{f}_{m}\right|^{2}$, which is the element of the $m_{t h}$ row and $m_{t h}$ column of the diagonal matrix $\frac{P_{R} \sigma_{R D}^{2}}{K P_{S} \sigma_{S R}^{2}+1} \mathbf{V}$, is much smaller than 1 (since $\left|\tilde{f}_{m}\right|^{2}$ has a unit variance). Hence, 
$\frac{P_{R} \sigma_{R D}^{2}}{K P_{S} \sigma_{S R}^{2}+1}\left|\tilde{f}_{m}\right|^{2}$ can be approximated by its mean value, which is $\frac{P_{R} \sigma_{R D}^{2}}{K P_{S} \sigma_{S R}^{2}+1}$. By plugging (52) into (51), we get

$E\left\{\tilde{\mathbf{n}}_{Z F} \tilde{\mathbf{n}}_{Z F}^{H} \mid \mathbf{H}, \mathbf{F}\right\} \approx\left(\frac{P_{R} \sigma_{R D}^{2}}{K P_{S} \sigma_{R D}^{2}+1}+1\right)\left(\mathbf{H V H} \mathbf{H}^{H}\right)^{-1}$.

Consequently, the approximate post-processing SNR for the $k_{t h}$ source is given by

$$
\begin{aligned}
& \gamma_{k}^{Z F} \approx \frac{\frac{P_{S} \sigma_{S R}^{2} P_{R} \sigma_{R D}^{2}}{K P_{S} \sigma_{S R}^{2}+1}}{\left(\frac{P_{R} \sigma_{R D}^{2}}{K P_{S} \sigma_{S R}^{2}+1}+1\right)\left[\left(\mathbf{H}^{H} \mathbf{V H}\right)^{-1}\right]_{k k}} \\
& \stackrel{(c)}{=} \bar{\gamma} \mathbf{h}_{k}^{H}\left(\mathbf{V}-\mathbf{V} \mathbf{H}_{(-k)}\left(\mathbf{H}_{(-k)}^{H} \mathbf{V H}\right.\right. \\
&\left.(-k))^{-1} \mathbf{H}_{(-k)}^{H} \mathbf{V}\right) \mathbf{h}_{k},
\end{aligned}
$$

where $\mathbf{h}_{k}$ is the $k_{t h}$ column of $\mathbf{H}, \mathbf{H}_{(-k)}$ is $\mathbf{H}$ with the $k_{t h}$ column removed and, furthermore, in $(c)$ we consider that the element of the $k_{t h}$ row and $k_{t h}$ column of the inverse of a square matrix $\mathbf{A}$, which we denote as $\left[\mathbf{A}^{-1}\right]_{k k}$, can be written as $\left[\mathbf{A}^{-1}\right]_{k k}=\left(\mathbf{A}_{k k}-\mathbf{a}_{k(-k)}^{H}\left(\mathbf{A}_{(-k,-k)}\right)^{-1} \mathbf{a}_{k(-k)}\right)^{-1}[31$, Eq. (8)], where $\mathbf{a}_{k(-k)}$ is the $k_{t h}$ column of $\mathbf{A}$ with the $k_{t h}$ entry removed and $\mathbf{A}_{(-k,-k)}$ is $\mathbf{A}$ with the $k_{t h}$ row and $k_{t h}$ column removed.

ii) $M_{\gamma, k}^{Z F}(s)$ : For the derivation of a closed-form of $M_{\gamma, k}^{Z F}(s)$, we follow a two-step procedure: 1) We first derive the MGF of $\gamma_{k}^{Z F}$ conditioned on $\mathbf{F}$, which we denote as $\left.M_{\gamma, k}^{Z F}(s \mid \mathbf{F}) .2\right)$ After deriving $M_{\gamma, k}^{Z F}(s \mid \mathbf{F}), M_{\gamma, k}^{Z F}(s)$ is obtained by marginalizing over $\mathbf{F}$ as $M_{\gamma, k}^{Z F}(s)=$ $E_{\mathbf{F}}\left\{M_{\gamma, k}^{Z F}(s \mid \mathbf{F})\right\}$.

1) Derivation of $M_{\gamma, k}^{Z F}(s \mid \mathbf{F})$ : By conditioning on $\mathbf{F}$, the channel matrix $\mathbf{F H}$ is equivalent to a Gaussian channel matrix $\mathbf{H}$ with correlation at the receive side, which is represented the matrix $\mathbf{V}$. By considering this, $\gamma_{k}^{Z F}$ has the same form as [32, Eq. (5)] and, consequently, $M_{\gamma, k}^{Z F}(s \mid \mathbf{F})$ is given by

$$
\begin{aligned}
& M_{\gamma, k}^{Z F}(s \mid \mathbf{F}) \stackrel{(d)}{\approx} \frac{1}{\operatorname{det}\left(s \bar{\gamma} \mathbf{V}+\mathbf{I}_{M}\right)} \\
& \times E_{\mathbf{H}_{(-k)} \mid \mathbf{F}}\left\{\frac{\operatorname{det}\left(\mathbf{H}_{(-k)}^{H} \mathbf{V} \mathbf{H}_{(-k)}\right)}{\operatorname{det}\left(\mathbf{H}_{(-k)}^{H} \mathbf{V}\left(s \bar{\gamma} \mathbf{V}+\mathbf{I}_{M}\right)^{-1} \mathbf{H}_{(-k)}\right)}\right\} \\
& \stackrel{s \bar{\gamma} \gg 1}{\approx} \frac{(s \bar{\gamma})^{K-1}}{\operatorname{det}\left(\mathbf{I}_{M}+s \bar{\gamma} \mathbf{V}\right)} E_{\mathbf{H}_{(-k)} \mid \mathbf{F}}\left\{\frac{\operatorname{det}\left(\mathbf{H}_{(-k)}^{H} \mathbf{V} \mathbf{H}_{(-k)}\right)}{\operatorname{det}\left(\mathbf{H}_{(-k)}^{H} \mathbf{I}_{M} \mathbf{H}_{(-k)}\right)}\right\},
\end{aligned}
$$

where in $(d)$ we use [32, Eq. (15)]. Now, according to [32, Eq. (17)], we have

$$
\begin{aligned}
& E_{\mathbf{H}_{(-k)} \mid \mathbf{F}}\left\{\frac{\operatorname{det}\left(\mathbf{H}_{(-k)}^{H} \mathbf{V} \mathbf{H}_{(-k)}\right)}{\operatorname{det}\left(\mathbf{H}_{(-k)}^{H} \mathbf{I}_{M} \mathbf{H}_{(-k)}\right)}\right\}= \\
& e l_{K-1}(\mathbf{V})(K-1) B(K-1, M-K+2),
\end{aligned}
$$

where $B(x, y)=\int_{0}^{\infty} \frac{t^{x-1}}{(1+t)^{x+y}} d t=\frac{(x-1) !(y-1) !}{(x+y-1) !}$ is the Beta function [27]. By plugging (56) into (55) and by using $\frac{\left|\tilde{f}_{m}\right|^{2}}{1+s \bar{\gamma}\left|\tilde{f}_{m}\right|^{2}} \approx(s \bar{\gamma})^{-1}$, which holds in the high-SNR regime $(s \bar{\gamma} \gg 1)$, we get

$$
M_{\gamma, k}^{Z F}(s \mid \mathbf{F}) \approx \frac{e l_{M-K+1}\left(\left(\mathbf{I}_{M}+s \bar{\gamma} \mathbf{V}\right)^{-1}\right)}{\left(\begin{array}{c}
M \\
K-1
\end{array}\right)}
$$

2) Derivation of $M_{\gamma, k}^{Z F}(s)$ : We have

$$
\begin{aligned}
M_{\gamma, k}^{Z F}(s) & =E_{\mathbf{F}}\left\{M_{\gamma, k}^{Z F}(s \mid \mathbf{F})\right\} \\
& \stackrel{(e)}{\approx} \frac{E_{\mathbf{F}}\left\{e l_{M-K+1}\left(\left(\mathbf{I}_{M}+s \bar{\gamma} \mathbf{V}\right)^{-1}\right)\right\}}{\left(\begin{array}{c}
M \\
K-1
\end{array}\right)},
\end{aligned}
$$

where in $(e)$ we use (57). Now, by considering that $f_{1}, \ldots, f_{M}$ are independent and identically distributed complex Gaussian random variables and by using [26, 3.352.4], we get

$$
\begin{aligned}
& E_{\mathbf{F}}\left\{e l_{M-K+1}\left(\left(\mathbf{I}_{M}+s \bar{\gamma} \mathbf{V}\right)^{-1}\right)\right\}= \\
& \left(\begin{array}{c}
M \\
M-K+1
\end{array}\right)\left[\frac{1}{s \bar{\gamma}} e^{\frac{1}{s \bar{\gamma}}} E_{1}\left(\frac{1}{s \bar{\gamma}}\right)\right]^{M-K+1} .
\end{aligned}
$$

By plugging (59) into (58) and by using (50), the proof of Proposition 3 is concluded.

A.4. Proof of Proposition 4: For $\bar{\gamma} \rightarrow \infty$ and by using the inequality $E_{1}(z)<e^{-z} \log \left(1+\frac{1}{z}\right)[27,5.1 .20]$ and the fact that $\log (c \bar{\gamma}) \simeq \log (\bar{\gamma})$ for $c>0$ and $\log (\bar{\gamma}) \gg \log (\log (\bar{\gamma}))$, as $\bar{\gamma} \rightarrow \infty$, (13) becomes

$$
S E R_{Z F} \lesssim \frac{c_{1}}{3}\left[c_{2}^{-(M-K+1)}+2 c_{3}^{-(M-K+1)}\right] \bar{\gamma}^{-(M-K+1)},
$$

which concludes the proof of Proposition 4.

A.5. Proof of Proposition 5: We again consider the following two steps, as in the proof of Proposition 3 for the $\mathrm{ZF}$ detector: i) We derive an approximate expression for the postprocessing Signal-to-Interference plus Noise Ratio (SINR) per source, which we denote as $\gamma_{k}^{M M S E}$. ii) Based on i), we obtain an approximate expression of the MGF of the derived postprocessing SINR, which we denote as $M_{\gamma, k}^{M M S E}(s)$, from which the SER per source of the MMSE detector can be obtained as [25, Eq. (33)]

$$
S E R_{M M S E} \approx c_{1}\left[\frac{1}{3} M_{\gamma, k}^{M M S E}\left(c_{2}\right)+\frac{2}{3} M_{\gamma, k}^{M M S E}\left(c_{3}\right)\right] .
$$

i) $\gamma_{k}^{M M S E}$ : We first find the variance of $\tilde{n}_{k}^{M M S E}$, conditioned on $\mathbf{H}$ and $\mathbf{F}$, which we denote as $\sigma_{M M S E, k \mid \mathbf{H}, \mathbf{F}}^{2}$. We have

$$
\begin{aligned}
\sigma_{M M S E, k \mid \mathbf{H}, \mathbf{F}}^{2} & =E\left\{\left(\tilde{n}_{k}^{M M S E}\right)^{2}\right\}-\left[E\left\{\tilde{n}_{k}^{M M S E}\right\}\right]^{2} \\
& \stackrel{(g)}{\approx} \frac{P_{S} \sigma_{S R}^{2} P_{R} \sigma_{R D}^{2}}{K P_{S} \sigma_{S R}^{2}+1} \\
& \times\left[\mathbf{g}_{M M S E, k}^{H} \mathbf{F} \mathbf{h}_{k}-\left(\mathbf{g}_{M M S E, k}^{H} \mathbf{F} \mathbf{h}_{k}\right)^{2}\right]
\end{aligned}
$$

where in $(g)$ we use (52). Consequently, based on (19) and 
after some algebraic manipulations, we have

$$
\begin{aligned}
\gamma_{k}^{M M S E} & \approx \frac{\left(\mathbf{g}_{M M S E, k}^{H} \mathbf{F} \mathbf{h}_{k}\right)^{2}}{\left[\mathbf{g}_{M M S E, k}^{H} \mathbf{F} \mathbf{h}_{k}-\left(\mathbf{g}_{M M S E, k}^{H} \mathbf{F} \mathbf{h}_{k}\right)^{2}\right]} \\
& =\mathbf{h}_{k}^{H} \mathbf{F}^{H}\left(\mathbf{F} \mathbf{H}_{(-k)} \mathbf{H}_{(-k)}^{H} \mathbf{F}^{H}+\frac{1}{\bar{\gamma}} \mathbf{I}_{M}\right)^{-1} \mathbf{F} \mathbf{h}_{k} .
\end{aligned}
$$

ii) $M_{\gamma, k}^{M M S E}(s)$ : The approximate closed-form expression for $M_{\gamma, k}^{M M S E}(s)$ will be derived in a two-step procedure, as in the case of the ZF detector: 1) We first derive the MGF of $\gamma_{k}^{M M S E}$ conditioned on $\mathbf{F}$, which we denote as $\left.M_{\gamma, k}^{M M S E}(s \mid \mathbf{F}) .2\right)$ After deriving $M_{\gamma, k}^{M M S E}(s \mid \mathbf{F}), M_{\gamma, k}^{Z F}(s)$ is obtained by marginalizing over $\mathbf{F}$ as $M_{\gamma, k}^{M M S E}(s)=$ $E_{\mathbf{F}}\left\{M_{\gamma, k}^{M M S E}(s \mid \mathbf{F})\right\}$.

1) Derivation of $M_{\gamma, k}^{M M S E}(s \mid \mathbf{F})$ : We again note that by conditioning on $\mathbf{F}$ the channel matrix $\mathbf{F H}$ is equivalent to a Gaussian channel matrix $\mathbf{H}$ with receive correlation, which is represented by the matrix $\mathbf{V}$. By considering this, $\gamma_{k}^{M M S E}$ has the same form as [32, Eq. (9)] and, consequently, $M_{\gamma, k}^{M M S E}(s \mid \mathbf{F})$ is given by

$$
\begin{aligned}
& M_{\gamma, k}^{M M S E}(s \mid \mathbf{F}) \stackrel{(h)}{\approx} \frac{1}{\operatorname{det}\left(\mathbf{I}_{M}+s \bar{\gamma} \mathbf{V}\right)} \times \\
& E_{\mathbf{H}_{(-k)}}\left\{\frac{\operatorname{det}\left(\mathbf{I}_{K-1}+\mathbf{H}_{(-k)}^{H}(\bar{\gamma} \mathbf{V}) \mathbf{H}_{(-k)}\right)}{\operatorname{det}\left(\mathbf{I}_{K-1}+\mathbf{H}_{(-k)}^{H}\left(\frac{1}{\bar{\gamma}} \mathbf{V}^{-1}+s \mathbf{I}_{M}\right)^{-\mathbf{1}} \mathbf{H}_{(-k)}\right)}\right\} \\
& \stackrel{s \bar{\gamma} \gg 1}{\approx} \frac{1}{\operatorname{det}\left(\mathbf{I}_{M}+s \bar{\gamma} \mathbf{V}\right)} \\
& \times E_{\mathbf{H}_{(-k)}}\left\{\frac{\operatorname{det}\left(\mathbf{H}_{(-k)}^{H}(\bar{\gamma} \mathbf{V}) \mathbf{H}_{(-k)}\right)}{\operatorname{det}\left(\mathbf{I}_{K-1}+\frac{1}{s} \mathbf{H}_{(-k)}^{H} \mathbf{H}_{(-k)}\right)}\right\}
\end{aligned}
$$

where in $(h)$ we use [32, Eq. (24)]. Now, according to [32, Eq. (25)], we have

$$
\begin{aligned}
& E_{\mathbf{H}_{(-k)}}\left\{\frac{\operatorname{det}\left(\mathbf{H}_{(-k)}^{H}(\bar{\gamma} \mathbf{V}) \mathbf{H}_{(-k)}\right)}{\operatorname{det}\left(\mathbf{I}_{K-1}+\frac{1}{s} \mathbf{H}_{(-k)}^{H} \mathbf{H}_{(-k)}\right)}\right\} \stackrel{(i)}{=} \\
& (K-1) e l_{K-1}(\bar{\gamma} \mathbf{V}) s^{K-1} e^{s} \\
& \times \sum_{k=0}^{K-2}(-1)^{k}\left(\begin{array}{c}
K-2 \\
k
\end{array}\right) E_{M-K+3+k}(s),
\end{aligned}
$$

where in $(i)$ we use [34, Eq. (6.21)]. By plugging (65) into (64) and by considering that $\frac{\left|\tilde{f}_{m}\right|^{2}}{1+s \bar{\gamma}\left|\tilde{f}_{m}\right|^{2}} \approx(s \bar{\gamma})^{-1}(s \bar{\gamma} \gg 1)$, we get

$$
\begin{aligned}
M_{\gamma, k}^{M M S E}(s \mid \mathbf{F}) & \approx(K-1) e l_{M-K+1}\left(\left(\mathbf{I}_{M}+s \bar{\gamma} \mathbf{V}\right)^{-1}\right) e^{s} \\
& \times \sum_{k=0}^{K-2}(-1)^{k}\left(\begin{array}{c}
K-2 \\
k
\end{array}\right) E_{M-K+3+k}(s)
\end{aligned}
$$

2) Derivation of $M_{\gamma, k}^{M M S E}(s)$ : We have

$$
\begin{aligned}
M_{\gamma, k}^{M M S E}(s) & =E_{\mathbf{F}}\left\{M_{\gamma, k}^{M M S E}(s \mid \mathbf{F})\right\} \stackrel{(j)}{\approx}(K-1) \\
& \times\left(\begin{array}{c}
M \\
M-K+1
\end{array}\right)\left[\frac{1}{s \bar{\gamma}} e^{\frac{1}{s \bar{\gamma}}} E_{1}\left(\frac{1}{s \bar{\gamma}}\right)\right]^{M-K+1} \\
& \times e^{s} \sum_{k=0}^{K-2}(-1)^{k}\left(\begin{array}{c}
K-2 \\
k
\end{array}\right) E_{M-K+3+k}(s),
\end{aligned}
$$

where in $(j)$ we use (59) and (66). By plugging (67) into (61), the proof of Proposition 5 is concluded.

A.6. Proof of Proposition 6: For $\bar{\gamma} \rightarrow \infty$ and by using the inequality $E_{1}(z)<e^{-z} \log \left(1+\frac{1}{z}\right)[27,5.1 .20]$ and the fact that $\log (c \bar{\gamma}) \simeq \log (\bar{\gamma})$ for $c>0$ and $\log (\bar{\gamma}) \gg \log (\log (\bar{\gamma}))$, as $\bar{\gamma} \rightarrow \infty$, (21) becomes

$$
\begin{aligned}
S E R_{M M S E} & \lesssim \frac{c_{1}}{3}(K-1)\left(\begin{array}{c}
M \\
M-K+1
\end{array}\right) \\
& \times\left[C\left(c_{2}\right)+2 C\left(c_{3}\right)\right] \bar{\gamma}^{-(M-K+1)} .
\end{aligned}
$$

Hence, the proof of Proposition 6 is concluded.

\section{APPENDIX B}

B.1. Proof of Theorem 1: For analytical simplicity and without loss of generality, we can approximate the ratio $\frac{G_{M M S E}^{c o d}}{G_{Z E}^{c o d}}$ by taking the first terms of numerator and denominator of (26), which gives (69) at the top of the next page. The approximation of (69) comes a result of considering the Chernoff bound in the approximation of the Q-function, instead of (46). Intuitively thinking, the value of $\frac{G_{M M S E}^{c o d}}{G_{Z F}^{c o d}}$ can only be slightly affected by this consideration since it is a ratio of coding gains, where each coding gain is obtained by the same approximation of the Q-function. This makes (69) a valid approximation. In addition,

$$
\begin{aligned}
& e^{c_{2}} \sum_{k=0}^{K-2}(-1)^{k}\left(\begin{array}{c}
K-2 \\
k
\end{array}\right) E_{M-K+3+k}\left(c_{2}\right) \stackrel{(k)}{>} \\
& c_{2}^{K-1} \frac{\Gamma(K-1) \Gamma\left(\mathrm{M}-K+3+c_{2}\right)}{\Gamma\left(\mathrm{M}+2+c_{2}\right)},
\end{aligned}
$$

where in $(k)$ we use [27, 5.1.19].

By plugging the right-side part of the inequality of (70) into (69), we get an upper bound of $\frac{G_{M M S E}^{c o d}}{G_{Z F}^{c o d}}$, that is

$$
\begin{aligned}
\frac{G_{M M S E}^{c o d}}{G_{Z F}^{c o d}} & <\left.\frac{G_{M M S E}^{c o d}}{G_{Z F}^{c o d}}\right|_{U B} \\
& =\left[\frac{(M-K+1) ! \Gamma\left(M+2+c_{2}\right)}{M ! \Gamma\left(M-K+3+c_{2}\right)}\right]^{\frac{1}{M-K+1}} .
\end{aligned}
$$

A good approximation for the argument of the factorial or gamma function can be obtained from Stirling's approximation, that is [27]

$$
n ! \approx \sqrt{2 \pi n}\left(\frac{n}{e}\right)^{n}, \quad \Gamma(1+t) \approx \sqrt{2 \pi t}\left(\frac{t}{e}\right)^{t} .
$$




$$
\frac{G_{M M S E}^{c o d}}{G_{Z F}^{c o d}} \approx\left[(K-1)\left(\begin{array}{c}
M \\
M-K+1
\end{array}\right) e^{c_{2}} \sum_{k=0}^{K-2}(-1)^{k}\left(\begin{array}{c}
K-2 \\
k
\end{array}\right) E_{M-K+3+k}\left(c_{2}\right)\right]^{-\frac{1}{M-K+1}}
$$

By plugging (72) into (71), we get

$$
\left.\frac{G_{M M S E}^{c o d}}{G_{Z F}^{c o d}}\right|_{U B} \approx m\left(K, M, c_{2}\right)^{b(K, M)}
$$

where

$m\left(K, M, c_{2}\right)=\frac{\left(M+1+c_{2}\right)^{M+c_{2}+\frac{3}{2}}(M-K+1)^{M-K+\frac{3}{2}}}{M^{M+\frac{1}{2}}\left(M-K+2+c_{2}\right)^{M-K+c_{2}+\frac{5}{2}}}$,

$b(K, M)=\frac{1}{M-K+1}$.

Now, since the gap between the coding gain (and, consequently, the corresponding SER) of the MMSE and ZF detectors is non-vanishing and does not depend on the SNR in the high-SNR regime, as it can be observed from (69), and as it was also proved in [20] for non-relay channels, then we have

$$
\begin{aligned}
\frac{G_{M M S E}^{c o d}}{G_{Z F}^{c o d}}>1 & \Rightarrow \log \left(\frac{G_{M M S E}^{c o d}}{G_{Z F}^{c o d}}\right)>0 \\
& \Rightarrow \log \left(\left.\frac{G_{M M S E}^{c o d}}{G_{Z F}^{c o d}}\right|_{U B}\right)>0 \\
& \Rightarrow \log \left[m\left(K, M, c_{2}\right)\right]>0
\end{aligned}
$$

Finally, we assume that $Q \gg 1$, which means that $c_{2} \approx \frac{3}{2 Q}$.

i) Proving that $\left.\frac{G_{M M S E}^{c o d}}{G_{Z F}^{c o d}}\right|_{U B}$ is a monotonically increasing function of $K$ : We have

$$
\begin{aligned}
& \frac{d\left(\left.\frac{G_{M M S E}^{c o d}}{G_{Z F}^{c o d}}\right|_{U B}\right)}{d K}=\left.\frac{G_{M M S E}^{c o d}}{G_{Z F}^{c o d}}\right|_{U B} \frac{1}{M-K+1} \\
& \times\left[\frac{1}{(M-K+1)} \log \left[m\left(K, M, c_{2}\right]\right)+\frac{\frac{d\left(m\left(K, M, c_{2}\right)\right)}{d K}}{m\left(K, M, c_{2}\right)}\right] .
\end{aligned}
$$

Hence, considering (75), to prove that (76) is a positive quantity it is adequate to prove that $\frac{d\left(m\left(K, M, c_{2}\right)\right)}{d K}$ is positive. We have:

$$
\frac{d\left(m\left(K, M, c_{2}\right)\right)}{d K}>0 \stackrel{(l)}{\Rightarrow}\left(1+c_{2}\right)\left(M-K+\frac{1}{2}\right)>0 \quad \text { true, }
$$

where in $(l)$ we use the inequality $\log (x) \leqslant x-1$ [35]. Consequently, i) is proven.

ii) Proving that $\left.\frac{G_{M M S E}^{c o d}}{G_{Z F}^{c o d}}\right|_{U B}$ is a monotonically decreasing function of $M$ : We have

$$
\begin{aligned}
& \frac{d\left(\left.\frac{G_{M M S E}^{c o d}}{G_{Z F}^{c o d}}\right|_{U B}\right)}{d M}=\left.\frac{G_{M M S E}^{c o d}}{G_{Z F}^{c o d}}\right|_{U B} \frac{1}{M-K+1} \\
& \times\left[-\frac{1}{(M-K+1)} \log \left[m\left(K, M, c_{2}\right]\right)+\frac{\frac{d\left(m\left(K, M, c_{2}\right)\right)}{d M}}{m\left(K, M, c_{2}\right)}\right] .
\end{aligned}
$$

Hence, by again considering (75), to prove that (78) is a negative quantity it is adequate to prove that $\frac{d\left(m\left(K, M, c_{2}\right)\right)}{d M}$ is negative. We have:

$$
\begin{aligned}
& \frac{d\left(m\left(K, M, c_{2}\right)\right)}{d M}<0 \\
& \stackrel{(m)}{\Rightarrow} 2+\frac{M-K+2.5+c_{2}}{M-K+2+c_{2}}+\frac{0.5}{M}-\frac{M+1.5+c_{2}}{M+1+c_{2}} \\
& -\frac{M-K+1.5}{M-K+1}-\frac{\left(M+1+c_{2}\right)(M-K+1)}{\left(M-K+2+c_{2}\right) M} \\
& >0 \quad \text { true, }
\end{aligned}
$$

where in $(m)$ we use the inequality $\log (x) \leqslant x-1[35]^{1}$. Consequently, ii) is proven.

iii) Proving that $\left.\frac{G_{M M S E}^{c o d}}{G_{Z F}^{c o d}}\right|_{U B}$ is a monotonically decreasing function of $Q$ : We have

$$
\begin{aligned}
\frac{d\left(\left.\frac{G_{M M S E}^{c o d}}{G_{Z F}^{c o d}}\right|_{U B}\right)}{d Q} & =b(K, M) m\left(K, M, c_{2}\right)^{b(K, M)-1} \\
& \times \frac{d\left[m\left(K, M, c_{2}\right)\right]}{d Q} .
\end{aligned}
$$

Hence, to prove that $(80)$ is a negative quantity it is adequate to prove that $\frac{d\left[m\left(K, M, c_{2}\right)\right]}{d Q}$ is negative. We have:

$$
\begin{aligned}
\frac{d\left[m\left(K, M, c_{2}\right)\right]}{d Q}<0 & \stackrel{(n)}{\Rightarrow}(M+1)(2 M-2 K+3) \\
& >\frac{3}{2 Q} \text { true }
\end{aligned}
$$

where in $(n)$ we use the inequality $1-\frac{1}{x} \leqslant \log (x)$ [35]. Consequently, iii) is proven, which concludes the proof of Theorem 1.

B.2. Proof of Theorem 2: From (35), we see that $D_{M L}\left(P_{R}\right)=D_{1}\left(P_{R}\right)+D_{2}\left(P_{R}\right)$, where

$$
\begin{aligned}
& D_{1}\left(P_{R}\right)=\frac{1}{\sigma_{S R}^{2}\left(P_{\text {budget }}-M P_{R}\right)}, \\
& D_{2}\left(P_{R}\right)=\frac{\log \left(1+\sigma_{R D}^{2} P_{R}\right)}{\sigma_{R D}^{2} P_{R}} .
\end{aligned}
$$

Hence, to prove that $D_{M L}\left(P_{R}\right)$ is convex, it is sufficient to prove that both $D_{1}\left(P_{R}\right)$ and $D_{2}\left(P_{R}\right)$ are convex since the sum of two convex functions in the same domain is also a convex function. This is because $D_{M L}^{\prime \prime}\left(P_{R}\right)=D_{1}^{\prime \prime}\left(P_{R}\right)+$ $D_{2}^{\prime \prime}\left(P_{R}\right)$, where $D_{M L}^{\prime \prime}\left(P_{R}\right), D_{1}^{\prime \prime}\left(P_{R}\right)$, and $D_{2}^{\prime \prime}\left(P_{R}\right)$ denote the second derivatives with respect to $P_{R}$ of $D_{M L}\left(P_{R}\right)$, $D_{1}\left(P_{R}\right)$, and $D_{2}\left(P_{R}\right)$, respectively. We have

$$
D_{1}^{\prime \prime}\left(P_{R}\right)=\frac{2 M^{2}\left(P_{\text {budget }}-M P_{R}\right)}{\sigma_{S R}^{2}\left(P_{\text {budget }}-M P_{R}\right)^{4}}>0,
$$

${ }^{1}$ We note that we have verified that the term $2+\frac{M-K+2.5+c_{2}}{M-K+2+c_{2}}+\frac{0.5}{M}-$ $\frac{M+1.5+c_{2}}{M+1+c_{2}}-\frac{M-K+1.5}{M-K+1}-\frac{\left(M+1+c_{2}\right)(M-K+1)}{\left(M-K+2+c_{2}\right) M}$ of (79) is positive by seeing $\begin{array}{ccc}M+1+c_{2} & M-K+1 & \left(M-K+2+c_{2}\right) M \\ \text { that it has no real root (by means of MATLAB). Hence, it maintains its positive }\end{array}$ sign for any value of $K, M$, and $Q$. 
since $P_{R} \in\left(0, \frac{P_{\text {budget }}}{M}\right)$, and

$$
\begin{aligned}
D_{2}^{\prime \prime}\left(P_{R}\right) & =\frac{2 \log \left(1+\sigma_{R D}^{2} P_{R}\right)}{\sigma_{R D}^{2} P_{R}^{3}}-\frac{3 \sigma_{R D}^{2} P_{R}+2}{P_{R}^{2}\left(1+\sigma_{R D}^{2} P_{R}\right)^{2}} \\
& \stackrel{(o)}{\geqslant} \frac{\left(\sigma_{R D}^{2}\right)^{2}}{\left(2+\sigma_{R D}^{2} P_{R}\right)\left(1+\sigma_{R D}^{2} P_{R}\right)^{2}}>0
\end{aligned}
$$

where in $(o)$ we use the inequality $\log (1+x) \geqslant \frac{2 x}{2+x}$ [35]. Consequently, we have proved that $D_{M L}\left(P_{R}\right)$ is a convex function.

B.3. Proof of Theorem 3: The proof of Theorem 3 consists of two steps: i) We prove that $S\left(P_{R}\right)$ is a concave function in $P_{R} \in\left(0, \frac{P_{\text {budget }}}{M}\right)$. ii) Having proven in the first step that $S\left(P_{R}\right)$ is concave in $P_{R} \in\left(0, \frac{P_{\text {budget }}}{M}\right)$, the second step consists of proving that $R\left(P_{R}\right)=\frac{\log \left(1+S_{\left.\left(P_{R}\right)\right)}\right.}{S\left(P_{R}\right)}$ is a convex function in $P_{R} \in\left(0, \frac{P_{\text {budget }}}{M}\right)$.

i) Proving that $S\left(P_{R}\right)$ is a concave function in $P_{R} \in$ $\left(0, \frac{P_{\text {budget }}}{M}\right)$ : The second derivative of $S\left(P_{R}\right)$ is given by

$$
S^{\prime \prime}\left(P_{R}\right)=-\frac{2\left(\sigma_{S R}^{2} P_{\text {budget }}+1\right)\left(\sigma_{R D}^{2} P_{\text {budget }}+M\right)}{\left(K P_{S} \sigma_{S R}^{2}+P_{R} \sigma_{R D}^{2}+1\right)^{3}}<0 .
$$

ii) Proving that $R\left(P_{R}\right)=\frac{\log \left(1+S\left(P_{R}\right)\right)}{S\left(P_{R}\right)}$ is a convex function in $P_{R} \in\left(0, \frac{P_{\text {budget }}}{M}\right):$ By recalling (84), which proves that the function $\frac{\log (1+x)}{x}, x>0$, is a convex function and by considering Lemma 1 and that $S\left(P_{R}\right)$ is a concave function in $P_{R} \in\left(0, \frac{P_{\text {budget }}}{M}\right)$, it follows that $R\left(P_{R}\right)=\frac{\log \left(1+S\left(P_{R}\right)\right)}{S\left(P_{R}\right)}$ is a convex function in $P_{R} \in\left(0, \frac{P_{\text {budget }}}{M}\right)$. The value of $P_{R}$, $P_{R_{\text {sol }}}$, that minimizes $R\left(P_{R}\right)$ can be found by taking the first derivative of $S\left(P_{R}\right)$ and setting it equal to 0 to find the root that falls in $P_{R} \in\left(0, \frac{P_{\text {budget }}}{M}\right)$. This leads to (41), which concludes the proof of Theorem 3 .

\section{REFERENCES}

[1] R. Ahlswede, C. Ning, S. Y. R. Li, and L. R. W. Yeung, "Network Information Flow", IEEE Trans. Inf. Theory, vol. 46, no. 4, pp. 1204-1212, July 2000

[2] S. Catti et.al., "XOR's in the air: Practical Wireless Network Coding", IEEE/ACM Trans. Networking, vol. 16, no. 3, pp. 497-510, June 2008.

[3] Q. Zhou, L. Young, F. C. M. Lau, B. Vucetic, "Decode-and-Forward Two-Way Relaying with Network Coding and Opportunistic Relay Selection” IEEE Trans. Commun., vol. 58, no. 11, pp. 3070-3076, June 2010.

[4] P. Popovski and H. Yomo, "Wireless network coding by amplify-andforward for bi-directional traffic flows", IEEE Commun. Letters, vol. 11, no. 1, pp. 16-18, Jan. 2007.

[5] T. Akino, P. Popovsi, and V. Tarokh., "Optimized constellations for twoway wireless relaying with physical network coding", IEEE Journal Sel. Areas. On Commun., vol. 27, no. 5, pp. 773-787, June. 2009.

[6] W. Lili and C. Wen, "Compute-and-Forward Network Coding Design over Multi-Source Multi-Relay Channels", IEEE Trans. Wireless Commun., vol. 11, no. 9, pp. 3348-3357, Sep. 2012.

[7] A. Raja and P. Viswanathan, "Compress-and-forward scheme for a relay network: Approximate optimality and connection to algebraic flows", IEEE ISIT Intern. Symp., pp. 1698-1702.

[8] S. Catti, S. Gollacota, and D. Katabi, "Embracing wireless interference: Analog network coding", ACM SIGCOMM, 2007.

[9] I. Maric, A. Goldsmith, and M. Medard, "Analog Network Coding in the High-SNR Regime", IEEE Wireless Netw. Coding Conf., June 2010.
[10] Y. Yang et. al., "Relay technologies for WiMax and LTE-advanced mobile systems", IEEE Commun. Magazine, vol. 47, no. 10, pp. 100105, Oct. 2009.

[11] D. Q. Chen, K. Azarian, and J. N. Laneman, "A case for amplify-forward relaying in the block-fading multiple-access channel", IEEE Trans. Inf. Theory, vol. 54, no. 8, pp. 3728-3733, Aug. 2008.

[12] M. Badr and J. C. Belfiore, "Distributed space time codes for the amplify-and-forward multiple-access relay channels", IEEE ISIT Intern. Symp., pp. 2543-2547.

[13] S. A. Jafar, K. S. Gomadam, and C. C. Huang, "Duality and rate optimization for multiple access and broadcast channels with amplifyand-forward relays", IEEE Trans. Inf. Theory, vol. 53, no. 10, pp. 3350-3370, Oct. 2007

[14] Z. G. Ding, T. Ratnarajah, and K. K. Leung, "On the study of network coded AF transmission protocol for wireless multiple access channels", IEEE Trans. Wireless Commun., vol. 8, no. 1, pp. 118-123, Jan. 2009.

[15] J. Yindi and B. Hassibi, "Distributed Space-Time Coding in Wireless Relay Networks", IEEE Trans. Wireless Commun., vol. 5, no. 12, pp. 3524-3536, Dec. 2006.

[16] Z. Wang and G. B. Giannakis, "A Simple and General Parameterization Quantifying Performance in Fading Channels", IEEE Trans. Commun., vol. 51, no. 8, pp. 1389-1398, Aug. 2003.

[17] W. Guan and K. J. R. Liu, "Diversity Analysis of Analog Network Coding with Multi-User Interferences", IEEE Trans. Wireless Commun., vol. 12, no. 2, pp. 668-679, Feb. 2013.

[18] K. Ntontin, M. Di Renzo, A. Pérez-Neira, and C. Verikoukis, "Error rate analysis and optimal power allocation in multiple access relay channels with Analog Network Coding”, IEEE Intern. Conf. on Commun., June 2014.

[19] L. Song, "Relay Selection for Two-Way Relaying With Amplify-andForward Protocols", IEEE Trans. Veh. Tech.., vol. 60, no. 4, pp. 1954-1959, May 2011.

[20] Y. Jiang, M. K. Varanasi, and J. Li, "Performance Analysis of ZF and MMSE Equalizers for MIMO Systems: An In-Depth Study of the High SNR Regime", IEEE Trans. Inf. Theory, vol. 57, no. 4, pp. 2008-2026, April 2011.

[21] I. G. Macdonald, Symmetric Functions and Hall Polynomials, Oxford University Press, 1999.

[22] M. Hasna and M.-S. Alouini, "A Performance Study of Dual-Hop Transmissions With Fixed Gain Relays", IEEE Trans. Wireless Commun., vol. 12, no. 2, pp. 1963-1968, Nov. 2004.

[23] X. Zhu and R. Merch, "Performance Analysis of Maximum Likelihood Detection in a MIMO Antenna System", IEEE Trans. Wireless Commun., vol. 50, no. 2, pp. 187-191, Feb. 2002.

[24] G. Tarrico and E. Biglieri, "Exact Pairwise Error Probability of Space-Time Codes", IEEE Trans. Inf. Theory., vol. 48, no. 2, pp. 510-513, Feb. 2002.

[25] N. Kim, Y. Lee, and H. Park, "Performance Analysis of MIMO System with Linear MMSE Receiver", IEEE Trans. Wireless Commun., vol. 7, no. 11 , pp. 4474-4478, Nov. 2008.

[26] I. S. Gradshteyn and I. M. Ryzhik, Table of Integrals, Series, and Products, 7th edition. Academic, 2007.

[27] M. Abramowitz and I. A. Stegun, Handbook of Mathematical Functions with Formulas, Graphs, and Mathematical Tables, 9th edition. Dover, 1972.

[28] B. Raghothaman et. al., "System Architecture for a Cellular Network with Cooperative Mobile Relay", IEEE Veh. Tech. Conf., Sep. 2011.

[29] T. Elkourdi and O. Simeone, "Femtocell as a Relay: An Outage Analysis", IEEE Trans. Wireless Commun., vol. 10, no. 12, pp. 4204-4213, Dec. 2011.

[30] P. Jacob and A. S. Madhukumar, "Femto-relays: A power efficient coverage extension mechanism for femtocells", IEEE PIMRC, Sep. 2011.

[31] P. Li, D. Paul, R. Narasimhan, and J. Cioffi "On the Distribution of SINR for the MMSE MIMO Receiver and Performance Analysis", IEEE Trans. Inf. Theory, vol. 52, no. 1, pp. 271-286, Jan. 2006.

[32] M. Kiessling and Joachim Speidel, "Unifying Performance Analysis of Linear MIMO Receivers in Correlated Rayleigh Fading Environments", ISSSTA, Sydney, Australia, 2004.

[33] C. Zhong et. al., "Performance of Rayleigh-Product MIMO Channels with Linear Receivers", IEEE Trans. Wireless Commun., vol. 13, no. 4, pp. 2270-2281, April 2004.

[34] M. Kiessling, "Statistical Analysis and Transmit Prefiltering for MIMO Wireless Systems in Correlated Fading Environments", Ph. D. Thesis Dissertation, 2004.

[35] F. Topsøe, Some bounds for the logarithmic function, RGMIA Research Report Collection, vol. 7(2), no. 6, 2004. 
[36] S. Boyd and L. Vandenberghe, Convex Optimization. Cambridge University Press, 2004. 